For Publication in the CCCP Special Issue of the Astrophysical Journal Supplement Series

Preprint typeset using $\mathrm{L}_{\mathrm{A}} \mathrm{X}$ style emulateapj v. 11/10/09

\title{
A PAN-CARINA YSO CATALOG: INTERMEDIATE-MASS YOUNG STELLAR OBJECTS IN THE CARINA NEBULA IDENTIFIED VIA MID-INFRARED EXCESS EMISSION
}

Matthew S. Povich, ${ }^{1,2}$ Nathan Smith, ${ }^{3}$ Steven R. Majewski, ${ }^{4}$ Konstantin V. Getman, ${ }^{1}$ Leisa K. Townsley, ${ }^{1}$ Brian L. Babler, ${ }^{5}$ Patrick S. Broos, ${ }^{1}$ Rémy Indebetouw,${ }^{4}$ Marilyn R. Meade, ${ }^{5}$ Thomas P. Robitaille,,${ }^{6,7}$ Keivan G. Stassun, ${ }^{8,9}$ Barbara A. Whitney, ${ }^{5,10}$ Yoshinori Yonekura, ${ }^{11,12}$ \& Yasuo Fukui $^{13}$

For Publication in the CCCP Special Issue of the Astrophysical Journal Supplement Series

\section{ABSTRACT}

We present a catalog of 1439 young stellar objects (YSOs) spanning the $1.42 \mathrm{deg}^{2}$ field surveyed by the Chandra Carina Complex Project (CCCP), which includes the major ionizing clusters and the most active sites of ongoing star formation within the Great Nebula in Carina. Candidate YSOs were identified via infrared (IR) excess emission from dusty circumstellar disks and envelopes, using data from the Spitzer Space Telescope (the Vela-Carina survey) and the Two-Micron All Sky Survey. We model the 1-24 $\mu \mathrm{m}$ IR spectral energy distributions of the YSOs to constrain physical properties. Our Pan-Carina YSO Catalog (PCYC) is dominated by intermediate-mass $\left(2 \mathrm{M}_{\odot}<m \lesssim 10 \mathrm{M}_{\odot}\right)$ objects with disks, including Herbig Ae/Be stars and their less evolved progenitors. The $\widetilde{P} C Y C$ provides a valuable complementary dataset to the CCCP X-ray source catalogs, identifying 1029 YSOs in Carina with no X-ray detection. We also catalog 410 YSOs with X-ray counterparts, including 62 candidate protostars. Candidate protostars with X-ray detections tend to be more evolved than those without. In most cases, X-ray emission apparently originating from intermediate-mass, disk-dominated YSOs is consistent with the presence of low-mass companions, but we also find that X-ray emission correlates with cooler stellar photospheres and higher disk masses. We suggest that intermediate-mass YSOs produce X-rays during their early pre-main sequence evolution, perhaps driven by magnetic dynamo activity during the convective atmosphere phase, but this emission dies off as the stars approach the main sequence. Extrapolating over the stellar initial mass function scaled to the PCYC population, we predict a total population of $>2 \times 10^{4}$ YSOs and a present-day star formation rate (SFR) of $>0.008 \mathrm{M}_{\odot} \mathrm{yr}^{-1}$. The global SFR in the Carina Nebula, averaged over the past $\sim 5 \mathrm{Myr}$, has been approximately constant.

Subject headings: circumstellar matter - ISM: individual (NGC 3372) — stars: formation — stars: luminosity function, mass function — stars: pre-main sequence - X-rays: stars

\section{INTRODUCTION}

The Great Nebula in Carina (NGC 3372) is among the most active massive star-forming complexes in the Galaxy. Over 70 O-type stars ionize a giant H II region (Smith 2006a) and power a bipolar superbubble that

\footnotetext{
${ }^{1}$ Department of Astronomy and Astrophysics, The Pennsylvania State University, 525 Davey Laboratory, University Park, PA 16802

${ }^{2}$ NSF Astronomy and Astrophysics Postdoctoral Fellow; povich@astro.psu.edu

${ }^{3}$ Steward Observatory, University of Arizona, 933 North Cherry Avenue, Tucson, AZ 85721

${ }^{4}$ Department of Astronomy, University of Virginia, P. O. Box 400325, Charlottesville, VA 22904-4325

${ }^{5}$ Department of Astronomy, University of Wisconsin, $475 \mathrm{~N}$. Charter Street, Madison, WI 53706

${ }^{6}$ Harvard-Smithsonian Center for Astrophysics, 60 Garden Street, Cambridge, MA 02138

7 Spitzer Postdoctoral Fellow

8 Department of Physics and Astronomy, Vanderbilt University, Nashville, TN 37235

${ }^{9}$ Department of Physics, Fisk University, 1000 17th Avenue N., Nashville, TN 37208

${ }^{10}$ Space Science Institute, 3100 Marine Street, Suite A353, Boulder, CO 80303

${ }^{11}$ Department of Physical Science, Osaka Prefecture University, 1-1 Gakuen-cho, Sakai, Osaka 599-8531, Japan

12 Center for Astronomy, Ibaraki University, 2-1-1 Bunkyo, Mito, Ibaraki 310-8512, Japan

13 Department of Astrophysics, Nagoya University, Furo-cho, Chikusa-ku, Nagoya 464-8602, Japan
}

has erupted from a 100-pc scale giant molecular cloud complex (Smith et al. 2000). The Carina Nebula is often considered to be the nearest analog of extragalactic starburst regions $(d=2.3 \mathrm{kpc}$; Smith 2006b), and it is also the least obscured. Carina thus presents a rich target for the study of resolved young stellar populations evolving in an environment dominated by some of the most massive stars known. While numerous studies have targeted individual rich ionizing clusters in Carina (Feinstein et al. 1980; Massey \& Johnson 1993; DeGioia-Eastwood et al. 2001; Ascenso et al. 2007; Sana et al. 2010), global observations of the stellar populations across the complex are challenging. Due to its large angular size $\left(>2^{\circ} \times 1.5^{\circ}\right)$, it is difficult to achieve the requisite wide observational coverage of the Carina Nebula at high spatial resolution. Its position near the Galactic midplane $(b=-0.6)$ means that wide-field images at visual and infrared (IR) wavelengths are dominated by contaminating populations of unassociated stars. The Chandra Carina Complex Project (CCCP), a 1.2 Ms total X-ray integration covering a $1.42 \mathrm{deg}^{2}$ field in the center of the Carina Nebula, provides an unprecedented opportunity to overcome these observational challenges and study the young stellar populations from a global perspective, yet at the detailed level of resolving individual stars (Townsley et al. 2011).

X-ray observations of star-forming regions are highly 
sensitive to low-mass pre-main-sequence (PMS) stars ( $\mathrm{T}$ Tauri stars), which generate hard X-rays via convectively-driven magnetic flaring activity (Preibisch et al. 2005) and to OB stars that produce soft X-rays primarily through micro-shocks in their strong stellar winds (Lucy \& White 1980; Feldmeier et al. 1997). Both classical $\mathrm{T}$ Tauri stars with circumstellar disks and protostars with accreting envelopes are observed to be significantly less luminous in X-rays compared to diskless, weak-lined T Tauri stars (Stassun et al. 2004; Telleschi et al. 2007; Prisinzano et al. 2008). The CCCP observations are expected to be insensitive to intermediate-mass $\left(2 \mathrm{M}_{\odot}<m<10 \mathrm{M}_{\odot}\right)$ young stellar objects (YSOs), including Herbig Ae/Be stars with disks and their less evolved progenitors, which lack a known mechanism for the production of strong X-ray emission (Stelzer et al. 2006). This important stellar population traces recent and ongoing star formation in massive complexes like Carina. Because such stars have high bolometric luminosities and possess dusty disks and envelopes that reprocess the stellar radiation into strong mid-IR emission, surveys with the Spitzer Space Telescope are very sensitive to them (Koenig et al. 2008; Robitaille et al. 2008; Kang et al. 2009; Povich \& Whitney 2010, hereafter PW10). The entire Carina Nebula was included in the Spitzer Vela-Carina survey. This complementarity between the IR and X-ray observations motivates us to search for candidate YSOs throughout the Carina Nebula using Vela-Carina survey data in conjunction with supplementary IR datasets.

The Carina complex contains at least $6.7 \times 10^{5} \mathrm{M}_{\odot}$ in molecular gas mass (Grabelsky et al. 1988) but hosts few bright embedded IR sources or other signposts of ongoing massive star formation; hence historically the Carina Nebula has been regarded as a relatively evolved $\mathrm{H}$ II region, with a current star formation rate (SFR) that is negligible in comparison with its historical rate (Smith \& Brooks 2008, and references therein). Within the past decade, a new view of star formation in Carina has emerged, in which active star formation has migrated to the outer reaches of the nebula, in particular the region known as the South Pillars, where remnant molecular cloud material has been shredded and sculpted into myriad clumps, pillars, and elephant-trunk structures by stellar winds and radiation, the two dominant feedback mechanisms produced by massive stars during their lifetimes (Megeath et al. 1996; Smith et al. 2000; Rathborne et al. 2004; Smith \& Brooks 2007). Smith et al. (2010b, hereafter SP10) presented the first Spitzer imaging study of Carina using the Infrared Array Camera (IRAC; Fazio et al. 2004) and found over 900 candidate YSOs widely distributed throughout two large fields covering the South Pillars and a portion of the opposite wall of the southern superbubble lobe. Carina remains a highly active star-forming region, but it may be unusual among Galactic giant molecular glouds (GMCs) in the sense that the dominant mode of current star formation activity appears to be triggering by feedback from the local massive stellar population (SP10).

Our present study expands upon the Spitzer pointsource results presented by SP10 in several ways: (1) The Vela-Carina observations extend Spitzer coverage to the entire Carina Nebula, including the central ionizing clusters and regions to the North. The wide-field coverage enables us to analyze relatively empty "control" fields far from the Carina GMC and correct for contamination in our YSO sample from unassociated sources with similar mid-IR colors. (2) We incorporate $24 \mu \mathrm{m}$ photometry from the Multiband Imaging Photometer for Spitzer (MIPS; Rieke et al. 2004) to constrain the evolutionary stages and luminosities of individual YSOs better than what is possible with IRAC photometry alone. (3) We analyze the subsample of YSOs that match CCCP cata$\log$ sources to investigate the physical properties of candidate X-ray-emitting protostars and intermediate-mass PMS stars with disks.

In this paper, we present a highly-reliable catalog of 1,439 predominantly intermediate-mass YSOs throughout the Carina Nebula, selected via their IR excess emission. This Pan-Carina YSO Catalog (PCYC) complements and augments the CCCP X-ray catalog (Broos et al. 2011a). The data analysis steps used to construct the PCYC are summarized in $\S 2$, and the methodology used to model the physical properties of the YSOs is described in $\S 3$. In $\S 4$, we present the ensemble properties of the YSO population, in $\S 5$ we analyze the subset of our sample associated with X-ray emission, and in $\S 6$ we highlight five noteworthy YSO clusters and sub-groups and several interesting individual YSOs. We discuss our results in the context of the star formation history of the Carina Nebula and the physical mechanism(s) powering $\mathrm{X}$-ray emission from YSOs in $\S 7$. We summarize our conclusions in $\S 8$.

\section{DATA ANALYSIS AND YSO SAMPLE SELECTION}

\subsection{Observations}

The Vela-Carina survey (Spitzer Proposal ID 40791, PI S. R. Majewski), was modeled after the Galactic Legacy Infrared Mid-Plane Survey Extraordinaire (GLIMPSE; Benjamin et al. 2003). It used the 4 mid-IR bands of Spitzer/IRAC $(3.6,4.5,5.8$, and $8.0 \mu \mathrm{m})$ to image $86 \mathrm{deg}^{2}$ of the Galactic plane in a strip $2^{\circ}$ high in latitude over longitudes $255^{\circ}<l<295^{\circ}$ (Zasowski et al. 2009), an area than included the Carina complex. PSF-fitting photometry was performed using the GLIMPSE point-source extractor, a modified version of DAOPHOT (Stetson 1987) optimized for crowded fields with strong, varying background nebular emission. The GLIMPSE extractor produces two source lists, the $99.5 \%$-reliable Point Source Catalog and the more complete Point Source Archive. ${ }^{14}$ The depth of the Vela-Carina photometry is very similar to that of GLIMPSE, reaching [3.6] $\lesssim 15.5$ mag (less sensitive in regions of bright nebular emission). Both the Catalog and the Archive sources have been matched (bandmerged) with $J H K_{S}$ photometry from the TwoMicron All-Sky Survey Point Source Catalog (2MASS; Skrutskie et al. 2006), and the resulting source lists provide photometry in $7 \mathrm{IR}$ bands, from $1.2 \mu \mathrm{m}$ through $8.0 \mu \mathrm{m}$. We use primarily the highly-reliable Catalog for this work, as experience shows that this drastically reduces the number of false-positives, candidate YSOs that are generated as the result of systematic photometric errors. The Vela-Carina Catalog contains 60,515 point sources inside the CCCP survey area (Table 1 ). We do retrieve a few sources from the Archive that are matched to

14 For details of the data processing and products, go to http://irsa.ipac.caltech.edu/data/SPITZER/GLIMPSE/doc/glimpse1_dataproc 
bright candidate YSOs detected by the Midcourse Space Experiment $(M S X)$, as described in $\S 2.4$ below.

The CCCP catalog contains over 14,000 point sources detected by the Advanced CCD Imaging Spectrometer (ACIS; Garmire et al. 2003) of the Chandra X-ray Observatory. Broos et al. (2011a) matched the Vela-Carina Archive to the CCCP X-ray catalog and found 6,543 counterparts to CCCP sources. The Vela-Carina Cata$\log$ is a subset of the Archive, and provides mid-IR counterparts for 4,664 CCCP sources (Table 1).

The Carina Nebula was observed with Spitzer/MIPS during late July 2007 as part of program GO-30848 (MIPSCAR; P.I. N. Smith). The program consisted of a series of MIPS scans that covered most of the area of the star forming region but avoided the bright emission of the Homunculus nebula around $\eta$ Carinae. This yielded a large mosaic image of the $24 \mu \mathrm{m}$ emission that contains the entire CCCP survey area.

\subsection{Identification of Candidate YSOs}

YSOs possess dusty circumstellar disks and infalling envelopes that reprocess radiation from the central stars, producing characteristic IR excess emission. YSOs can be identified in broad-band photometric imaging observations via their IR colors or spectral energy distributions (SEDs). Our procedure for identifying candidate YSOs is adapted from Povich et al. (2009), SP10, and PW10. Here we outline the main steps used in constructing our highly-reliable catalog of candidate YSOs, and we summarize the number of sources found within the CCCP survey area for each step in Table 1. Using a $\chi^{2}$-minimization SED fitting tool (Robitaille et al. 2007), we fit reddened Castelli \& Kurucz (2004) stellar atmospheres (allowing $A_{V}$ to vary between 0 and 40 mag according to the extinction law of Indebetouw et al. 2005) to the SEDs of 210,742 sources in the VelaCarina Catalog located within a $\Delta l \times \Delta b=2.8 \times 2^{\circ}$ field, 54,155 of which fell within the CCCP survey area (Table 1). ${ }^{15}$ Only sources detected in $N_{\text {data }} \geq 4$ of the 7 Catalog bands $(\lambda=1-8 \mu \mathrm{m})$ were fit. To avoid biasing the fits for sources where the photometric uncertainties may have been underestimated in the Catalog, before fitting any models we conservatively reset the uncertainties to a minimum value of $10 \%$. We considered sources for which $\chi_{0}^{2} / N_{\text {data }} \leq 2\left(\chi_{0}^{2}\right.$ is the unreduced goodness-of-fit parameter of the best-fit model; SP10) to be well-fit by stellar atmosphere SEDs; among sources in the CCCP field that were fit with SED models, $93.4 \%$ were consistent with normally-reddened stellar photospheres (Table 1) and hence were removed from consideration for the PCYC.

We then filtered out sources with "marginal" IR excess emission, where the excess appears in only the single IRAC [5.8] or [8.0] band, using the SP10 color criteria, modified to de-redden the [3.6] - [4.5] colors of background stars viewed through the Carina cloud by up to $A_{V}=20$ mag. Using $[\lambda]$ to denote magnitudes in the various IRAC bands and $\delta\left(\left[\lambda_{i}\right]-\left[\lambda_{j}\right]\right)$ for the uncertainties on the colors computed from the (minimum 10\%) uncertainties on Catalog flux densities, the selection criteria

\footnotetext{
15 The full field analyzed is much larger than the CCCP field; regions of active star formation identified in this wider field will be the subject of a future paper.
}

were as follows:

$$
\begin{aligned}
{[3.6] } & -[4.5]>\delta([3.6]-[4.5])+E([3.6]-[4.5]) \\
\text { OR } \quad|[4.5]-[5.8]| & >\delta([4.5]-[5.8]) \\
\text { AND } \quad[5.8]-[8.0] & >\delta([5.8]-[8.0]) .
\end{aligned}
$$

The color excess used for the de-reddening was calculated as

$$
E([3.6]-[4.5])=A_{V} \frac{\left(\kappa_{3.6}-\kappa_{4.5}\right)}{\kappa_{V}}=0.0135 A_{V},
$$

where the $\kappa_{\lambda}$ are opacities given by the extinction law (Indebetouw et al. 2005). This step is crucial to distinguish intrinsically red objects from sources affected by potential systematic photometric errors (Povich et al. 2009); IRAC [5.8] and [8.0] are less sensitive to point sources and more affected by nebular emission compared to IRAC [3.6] and [4.5]. We discarded 1,923 of these "marginal" IR excess sources, leaving 1,646 "reliable" intrinsic IR excess sources, which form the basis for our sample of candidate YSOs (Table 1). Throwing out the majority of the possible IR excess sources in this manner may seem overly conservative, but we note that among the CCCP-matched subsample, only $1 / 3$ of sources were caught by this filter (Table 1). The Vela-Carina survey images are dominated by $\mathrm{K}$ - and M-type giants in the Galactic field that are not expected to emit X-rays (Rosner et al. 1995). If the majority of marginal IR excess sources were actually due to emission from disks, perhaps transitional disks with large inner holes, we would expect the fraction of sources rejected at this step among the X-ray detected subsample to be similar to, if not higher than, the fraction rejected from the full sample. Instead, the significantly lower frequency of marginal IR excess sources with X-ray counterparts affirms that the majority of these sources are unassociated field stars. The 213 CCCP-matched sources in Table 1 rejected on account of marginal IR excess are examined again by Povich et al. (2011, hereafter P11).

\subsection{MIPS 24 $\mu m$ Aperture Photometry}

Our data analysis is tailored to the goal of fitting YSO model SEDs from Robitaille et al. (2006, hereafter RW06) to the available IR photometry of each candidate YSO. Because the SEDs of highly embedded YSOs peak in the thermal IR near $100 \mu \mathrm{m}$, photometry at $\lambda>10 \mu \mathrm{m}$ is often required to constrain bolometric luminosities and to distinguish envelope-dominated (Stage 0/I) from diskdominated (Stage II) YSOs (RW06; Indebetouw et al. 2007). Following PW10, we measured MIPS $24 \mu \mathrm{m}$ flux densities using aperture photometry. We located the position of each candidate YSO and centered an extraction aperture of radius $3.5^{\prime \prime}$ and background annulus of inner and outer radii $7^{\prime \prime}$ and $13^{\prime \prime}$, respectively, on the MIPS $24 \mu \mathrm{m}$ mosaic of Carina. We extracted $24 \mu \mathrm{m}$ flux densities for each source, estimating the background level using the Daophot MMM algorithm (Stetson 1987). Our choice of extraction aperture required an aperture correc$\operatorname{tion}^{16}$ of 2.8 . The uncertainty introduced by the aperture correction is more than offset by the greater accuracy of

16
http://ssc.spitzer.caltech.edu/mips/mipsinstrumenthandbook/ 
Table 1

Summary of Vela-Carina Point Sources in CCCP Field

\begin{tabular}{lrrl}
\hline \hline \multicolumn{1}{c}{ Sources } & All & CCCP-matched & Description \\
\hline In Vela-Carina Catalog & 60,515 & 4,664 & Detected IRAC point sources, 99.5\% reliable \\
Fit with SED Models & 54,155 & 4,080 & Detected in $\geq 4$ of 7 2MASS+IRAC bands \\
Stellar Photospheres & 50,586 & 3,444 & Well-fit by stellar atmosphere SEDs \\
Possible IR Excess & 3,569 & 636 & Poorly-fit by stellar atmosphere SEDs \\
Marginal IR Excess & 1,923 & 213 & Excess in only IRAC [5.8] or [8.0] band \\
Reliable IR Excess & 1,646 & 423 & Intrinsically red objects \\
\hline In PCYC & 1,439 & 410 & YSO catalog, corrected for contamination \\
Stage 0/I & 247 & 62 & Dominant SED models: infalling envelopes \\
Stage II & 478 & 190 & Dominant SED models: optically thick disks \\
Stage III & 83 & 13 & Dominant SED models: optically thin disks \\
Ambiguous & 631 & 144 & Inconclusive SED model classification \\
\hline a Includes 3 Vela-Carina Archive sources with $M S X$ counterparts.
\end{tabular}

a Includes 3 Vela-Carina Archive sources with $M S X$ counterparts.

the local background determination and the ability to separate close sources (PW10).

PW10 analyzed MIPSGAL $24 \mu \mathrm{m}$ survey data (Carey et al. 2009) covering the M17 SWex IR dark cloud. The MIPSCAR observations are shallower than the MIPSGAL observations and affected by extremely bright nebular emission produced by warm dust in the Carina $\mathrm{H}$ II region. While $\sim 65 \%$ of the YSOs cataloged by PW10 were detected at $24 \mu \mathrm{m}$, the detection fraction is only $\sim 10 \%$ for the PCYC. We can, however, derive useful upper limits on $24 \mu \mathrm{m}$ flux densities in the majority of cases. Upper limits provide critical constraints for the SED fitting, since the RW06 models may predict variations of more than an order of magnitude in $24 \mu \mathrm{m}$ flux density even when the IRAC flux densities are well-measured. Detections and upper limits were defined as follows: The formal uncertainty $\sigma$ on the aperture photometry is generally dominated by the uncertainty on the photometric background determination but also takes into account photon counting statistics (including the contribution of the zodiacal background light). Taking $F^{\prime}$ to be the extracted flux density, sources for which $F_{24}^{\prime}<5 \sigma$ were considered undetected at $24 \mu \mathrm{m}$, and upper limits of $F_{24}<5 \sigma+F_{24}^{\prime}$ for $F_{24}^{\prime}>0$ and $F_{24}<5 \sigma$ for $F_{24}^{\prime} \leq 0$ were assigned. The results of our aperture photometry are presented in Table 2 , in which the $24 \mu \mathrm{m}$ quality flags (column 20) are set to 1 for detections and 3 for upper limits. No photometric information can be obtained for sources falling within regions of the $24 \mu \mathrm{m}$ mosaic where the nebular emission saturates (for example, in the vicinity of $\eta$ Car); these are given quality flags of 0 . For the $24 \mu \mathrm{m}$ detections we set the minimum uncertainty to $0.15 F_{24}$ to account for systematic uncertainties. One potential source of systematics is the mid-IR extinction law for $\lambda>10 \mu \mathrm{m}$, which appears to vary with environment. Recent observational work with Spitzer has found that, in molecular clouds, the absorption at $24 \mu \mathrm{m}$ is similar to, not less than, the absorption at $8 \mu \mathrm{m}$ (e.g. Flaherty et al. 2007). We therefore used the high-column-density $\left(A_{K} \geq 1\right)$ mid-IR extinction law of McClure (2009) when fitting the RW06 models to the SEDs of the candidate YSOs.

\subsection{MSX Detections of Luminous Candidate YSOs}

We incorporate $8-21 \mu \mathrm{m}$ photometry from the $M S X$ Galactic Plane Survey Point Source Catalog (Price et al. 2001) into our YSO sample in 2 ways: (1) Spatial correlation of $M S X$ sources with the candidate YSOs and
(2) visual identification of bright mid-IR sources within the CCCP field that were excluded from the Vela-Carina Catalog because they were either marginally resolved or saturated. Moderately saturated sources were included in the more complete Vela-Carina Point Source Archive, and we added $3 M S X$ sources with Archive matches to the sample. Only $11 \mathrm{PCYC}$ sources have $M S X$ counterparts (indicated in Table 2), but these include the most luminous YSOs in the cloud. Most of these objects were previously cataloged by Rathborne et al. (2004) or Smith \& Brooks (2007). The addition of up to $4 M S X$ datapoints provides strong constraints on the mid-IR SEDs along with replacements for Spitzer photometry suffering from saturation at 8.0 or $24 \mu \mathrm{m}$.

\subsection{Removal of Contaminating IR Excess Sources}

While it is dominated by YSOs, the sample of reliable IR excess sources (Table 1) may also contain the following types of contaminants: variable stars, dusty asymptotic giant branch (AGB) stars, unresolved planetary nebulae, and background galaxies (Robitaille et al. 2008; Povich et al. 2009). Although few in number, luminous AGB stars are the most important contaminants, as they can masquerade as massive YSOs. The majority of AGB stars have [8.0] $-[24]<2.2 \mathrm{mag}$, and the few extreme AGB stars that are redder are highly luminous and would be conspicuously bright (Whitney et al. 2008; Povich et al. 2009). Applying this color cut removed 1\% of sources. We correct for the remaining contamination by analyzing the spatial distribution of IR excess sources.

While YSOs associated with the Carina Nebula exhibit a high degree of clustering (SP10), contaminating sources, including foreground YSOs, are distributed uniformly. We used the nearest-neighbor group-finding algorithm described by Povich et al. (2009) to identify sources exhibiting a significant degree of clustering with respect to 3 "control" fields located outside of the CCCP field. The algorithm was tuned to be sensitive to groupings of $\geq 10$ sources. The control fields were $0.5 \times 0.5$ boxes centered at $(l, b)=(286.4,-0.9),(286.6,0.2)$, and $\left(288^{\circ} 3,0^{\circ} 1\right)$, containing a mean surface density of reliable IR excess sources of $\Sigma_{\text {con }}=200 \mathrm{deg}^{-2}$. The surface density of reliable IR excess sources inside the $1.42 \mathrm{deg}^{2}$ CCCP field is $\Sigma_{\mathrm{CCCP}}=1150 \mathrm{deg}^{-2}$ (Table 1), hence the expected contamination fraction from sources unassociated with the Carina Nebula is $\Sigma_{\text {con }} / \Sigma_{\text {CCCP }}=17 \%$. After removing sources from the sample that did not satisfy the clustering criterion, the source density in the final 
PCYC is $\Sigma_{\mathrm{CCCP}}^{\mathrm{corr}}=1000 \mathrm{deg}^{-2}$, indicating a residual contamination level of $\Sigma_{\mathrm{CCCP}}^{\mathrm{corr}}-\left(\Sigma_{\mathrm{CCCP}}-\Sigma_{\mathrm{con}}\right)=50 \mathrm{deg}^{-2}$, or $5 \%$.

\section{MODEL-BASED YSO CHARACTERIZATION AND CLASSIFICATION}

Basic IR photometric data for the 1439 YSOs in the PCYC are presented in Table 2. Using the Robitaille et al. (2007) SED fitting tool, we fit YSO models from RW06 to the available photometry of each source. We allowed the fitting tool to accept a range of distances of $2.3 \pm 0.05 \mathrm{kpc}$, guided by the well-established distance to $\eta$ Car from expansion measurements of the Homunculus nebula (Smith 2006b) and by the assumption that the depth of the Carina Nebula along the line of sight is not greater than its $\sim 50 \mathrm{pc}$ diameter observed on the sky. We define the set $i$ of well-fit models for each YSO according to $\chi_{i}^{2}-\chi_{0}^{2} \leq 2 N_{\text {data }}$, and assign a $\chi^{2}$-weighted, normalized probability $P_{i}$ to each model (Povich et al. 2009). This enables us to construct probability distributions for the model parameters and thereby constrain physical properties such as luminosity, mass, and accretion rate. In general, the parameters are not normally distributed, so the physical properties for individual sources must be interpreted with care. We present results from the model fitting to the PCYC sources in columns (2)-(8) of Table 3. For each YSO, we list characteristic values of 3 parameters along with corresponding uncertainties. The characteristic values for the stellar parameters mass $\left(X=M_{\star}\right)$ and bolometric luminosity $\left(X=L_{\mathrm{bol}}\right)$ are calculated as the probability-weighted means of the parameter distributions,

$$
\langle X\rangle=\sum P_{i} X_{i}
$$

Circumstellar parameters in the RW06 models, such as envelope accretion rate $\left(Y=\dot{M}_{\text {env }}\right)$, span many orders of magnitude. We define the characteristic circumstellar parameters as the medians of the parameter distributions,

$$
\langle Y\rangle=\mu_{1 / 2}(Y),
$$

because this approach is less affected by any extreme outlying values returned by the fitting procedure. For both stellar and circumstellar parameters, we compute the $1 \sigma$ uncertainty $\sigma(X, Y)$ as the standard deviation on the characteristic parameter value $\langle X, Y\rangle$.

The YSO models are divided into evolutionary stages that parallel the well-known empirical T Tauri classification system based on IR colors or spectral indices: Stage 0/I YSOs are still embedded in their infalling, natal envelopes; Stage II YSOs have optically thick circumstellar disks; Stage III YSOs have optically thin disks (RW06). We construct probability distributions of evolutionary stage for each YSO and classify a YSO as Stage 0/I, II, or III if $\sum P_{i}$ (Stage) $\geq 0.67$; we refer to the classification of sources not meeting this criterion as "Ambiguous" (Povich et al. 2009). We prefer the RW06 stage classification system to the traditional $\mathrm{T}$ Tauri class system because (1) it makes the model-dependent nature of the taxonomy explicit rather than implicit, (2) the incorporation of Ambiguous classifications acknowledges the limitations of imposing distinct categories on a continuous evolutionary sequence, and (3) a model-based system provides physical insight into intermediate- and highmass YSOs for which a classification based on the empirical colors of low-mass $\mathrm{T}$ Tauri stars may not be meaningful. The distribution of stage classifications across the PCYC is presented in Table 1, and the classification for each YSO is given in Table 3 (column 8).

The 410 YSOs detected in X-rays are identified in Table 3 by CCCP catalog name (column 9). Feigelson et al. (2011, hereafter F11) divided the CCCP field into 4 broad regions (A, B, C, and D) and also identified 20 X-ray clusters and 31 smaller groups. We assign every PCYC source to one of these regions and possibly also to one of the clusters/groups according to the region/cluster/group membership of the nearest CCCP catalog source (columns 10 and 11 of Table 3).

The PCYC (Tables 2 and 3) is a compilation of individual candidate YSOs in Carina that we hope will provide a rich dataset for future follow-up studies. For the present work, we are concerned primarily with the ensemble properties of the population. The large sample size provides robust statistics to compensate for potentially large uncertainties on individual YSOs whose physical properties, such as stellar mass and bolometric luminosity, may not be well-constrained by the RW06 models.

\section{GLOBAL PROPERTIES OF THE CARINA YSO POPULATION \\ 4.1. Spatial Distribution}

The positions of sources in the YSO catalog are plotted on an IRAC $8.0 \mu \mathrm{m}$ mosaic image from the Vela-Carina survey in Figure 1. While YSOs are distributed widely throughout the CCCP field, the population generally traces the broad North-South stellar "backbone" joining the major ionizing clusters Trumpler ( $\mathrm{Tr}$ ) 14 and 16 with the Collinder (Cr) 228/South Pillars region (Townsley et al. 2011, F11). Contours of the major molecular clouds traced in $\mathrm{CO}$ emission and the locations of 8 dense $\mathrm{C}^{18} \mathrm{O}$ molecular cloud cores (Yonekura et al. 2005) are also plotted in Figure 1. While the spatial distribution of YSOs throughout the Carina Nebula exhibits highly complex structure, with clustering on multiple spatial scales, a general pattern is readily discernible. The majority of YSOs are located inside H II region cavities near, but less frequently within, the boundaries of dense molecular clouds and the ends of pillars.

Another view of the PCYC is presented in Figure 2, in which a Vela-Carina $3.6 \mu \mathrm{m}$ mosaic is presented with YSOs color-coded by mass. YSOs in 3 mass ranges roughly corresponding to the mass ranges of Herbig Be stars, Herbig Ae stars, and T Tauri stars are colored red, yellow, and green, respectively. Density contours of CCCP sources from F11 depict all of the major clusters as well as numerous smaller stellar groupings. The largest concentration of intermediate-mass YSOs is found not in the South Pillars, but in Tr 14 itself. Numerous sub-clusters of Tr 16 (F11) also have associated YSOs.

Several caveats regarding the Spitzer point-source detections must be kept in mind when analyzing the PCYC. The strong and variable nebular emission across the Carina Nebula, especially at 8.0 and $24 \mu \mathrm{m}$, creates complicated, position-dependent variations in the sensitivity limit for mid-IR point-source detections. In addition, the IRAC images are confusion-limited in the dense clus- 


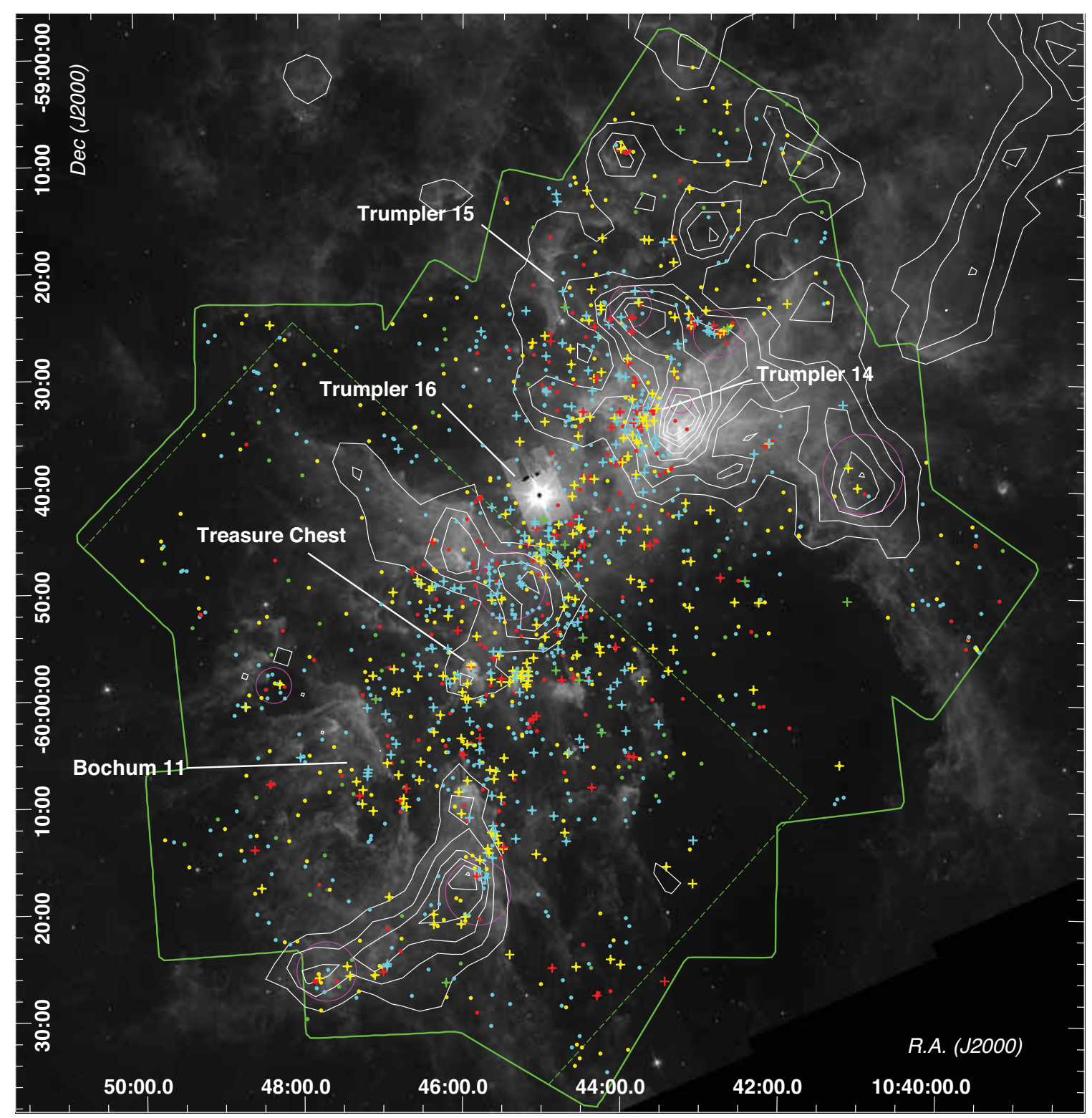

Figure 1. Spitzer/IRAC $8.0 \mu \mathrm{m}$ mosaic image with positions of the 1439 YSOs in the Pan-Carina YSO Catalog (PCYC) overplotted. Colors identify YSO evolutionary stages: red=Stage 0/I, yellow=Stage II, green=Stage III, cyan=ambiguous. YSOs with X-ray counterparts are marked by crosses. Contours are CO integrated intensity over the velocity range $V_{\mathrm{LSR}}=-30 \mathrm{~km} \mathrm{~s}^{-1}$ to $-10 \mathrm{~km} \mathrm{~s}^{-1}$, and the large magenta circles mark $\mathrm{C}^{18} \mathrm{O}$ molecular cores (Yonekura et al. 2005). The solid green outline is the boundary of the CCCP survey area, while the dashed green lines enclose the area where the South Pillars observation of SP10 overlaps the CCCP. The bright, saturated star near the image center is $\eta$ Car. Five well-known massive star clusters discussed in the text are labeled.

ter centers. The PCYC is very incomplete near $\eta$ Car, a highly saturated Spitzer source visible at the center of Figures 1 and 2, and in the Treasure Chest, a very young, embedded cluster (Smith et al. 2005), where only 3 candidate YSOs are detected due to extreme crowding and nebulosity. Other potential areas of sensitivity problems include the crowded center of $\operatorname{Tr} 14$ and the ionization front between $\operatorname{Tr} 14$ and the Car I molecular cloud core to the west (Yonekura et al. 2005; Ascenso et al. 2007) where the diffuse mid-IR nebular emission is bright. Nevertheless, the dearth of active star formation within the Car I cloud core appears to be real, not simply a sensitivity effect.

All of the Spitzer YSO sub-clusters cataloged by SP10 are apparent in Figures 1 and 2. In addition, we detect 3 new, tight clusters or groups of $\sim 10$ YSOs in the
Northwest corner of the CCCP area, 2 of which are associated with significant overdensities of X-ray sources (F11). We discuss these new YSO clusters in more detail in $\S 6$. YSOs are also detected embedded in the ends of most dust pillars, both in the South Pillars and elsewhere in the Carina Nebula. Some of these are the driving sources of the Herbig-Haro jets found in Hubble Space Telescope images (Smith et al. 2010a). YSOs are also associated with several of the candidate proplyds identified by Smith et al. (2003), specifically proplyds 104405.4592940 (PCYC 429), 104619.7-595044 (1126), 104632.9600353 (1173), and 104519.3-594423 (842 and 841). All of the YSOs associated with proplyds have either Stage $0 / \mathrm{I}$ or Ambiguous classifications, and none are detected in X-rays. 


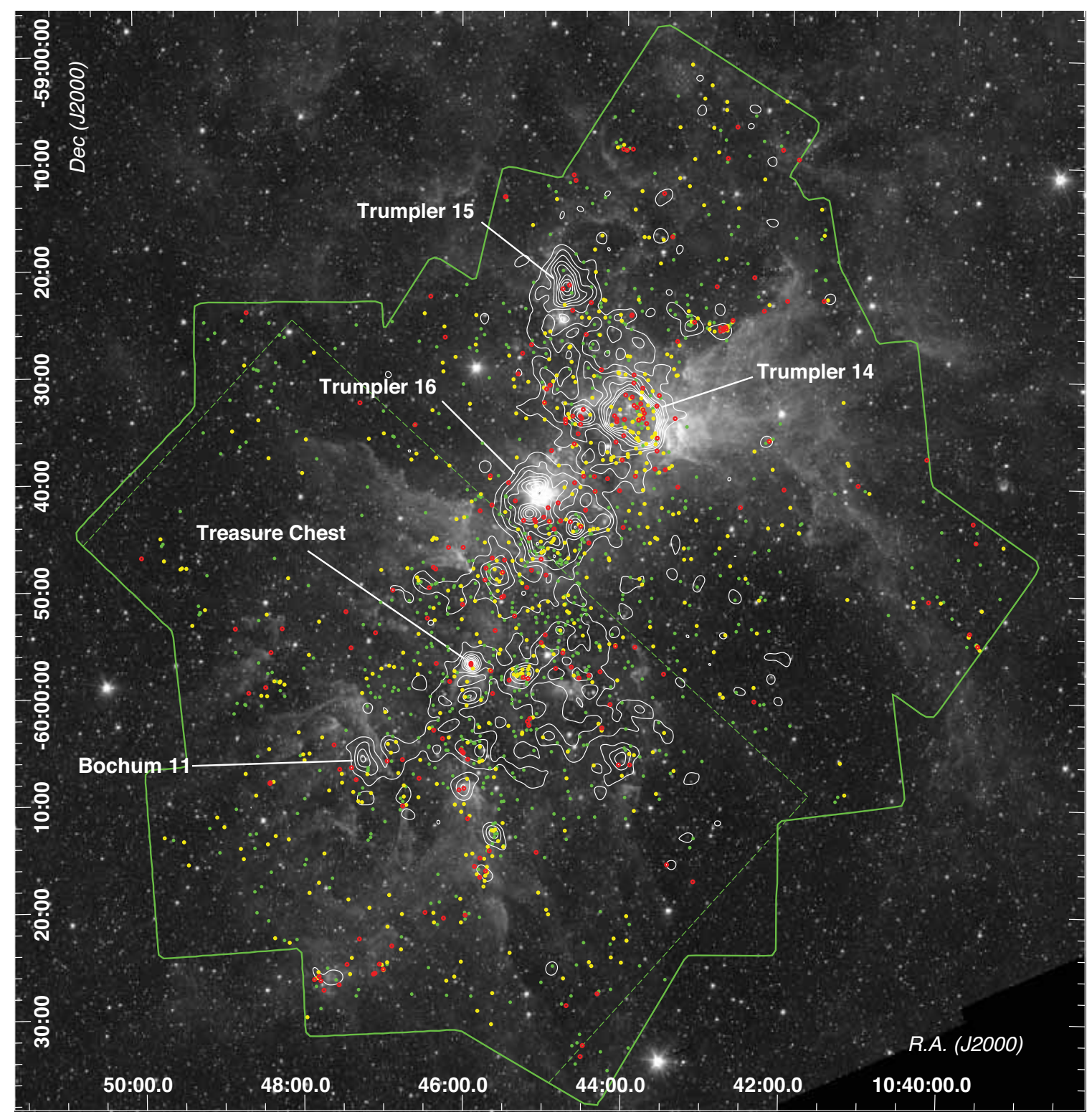

Figure 2. Spitzer-IRAC $3.6 \mu \mathrm{m}$ mosaic image with positions of the 1439 YSOs in the PCYC overplotted. Colors identify YSOs by mass range: red, $\left\langle M_{\star}\right\rangle \geq 3.1 \mathrm{M}_{\odot}$; yellow, $2 \mathrm{M}_{\odot} \leq\left\langle M_{\star}\right\rangle<3.1 \mathrm{M}_{\odot}$; green, $\left\langle M_{\star}\right\rangle<2 \mathrm{M}_{\odot}$. Contours are from $\mathrm{F} 11$ and show the surface density of X-ray sources. The green outlines and cluster labels are the same as in Figure 1.

\subsection{YSO Mass Function and Present-Day Star Formation Rate}

Summing the individual probability distributions of $M_{\star}$ defined by the set of models fit to each YSO, we construct the YSO mass function (YMF; Shepherd et al. 2007; Povich et al. 2009; Kang et al. 2009, PW10) for all 1439 YSOs in the PCYC (Figure 3). The YMF is defined similarly to the stellar initial mass function (IMF), $\Phi(\log m)=d N / d \log m$, where $d N$ is the number of stars in the (logarithmic) mass interval $(\log m, \log m+d \log m)$. The Carina YMF exhibits a power-law form $\Psi(m) \propto$ $m^{-\Gamma_{\mathrm{YMF}}}$ for $M_{c} \leq m<10 \mathrm{M}_{\odot}$, departing from the power-law due to incompleteness for $m<M_{p} \approx 3.1 \mathrm{M}_{\odot}$ (Figure 3a). The power-law slope $\Gamma_{\mathrm{YMF}}=3.2 \pm 0.3$ is significantly steeper than the standard Salpeter-Kroupa $\mathrm{IMF}$ slope $\Gamma_{\mathrm{IMF}}=1.3$ (Kroupa 2001). PW10 found a very similar $\mathrm{YMF}$ shape, with $\Gamma_{\mathrm{YMF}}=3.5 \pm 0.6$, for a sample of 488 YSOs in M17 SWex, a GMC com- plex extending $\sim 50 \mathrm{pc}$ outward from the bright Galactic H II region M17.

In Figure 3b, the YMF has been broken down by most probable evolutionary stage. This shows that the overall shape of the YMF is dominated by a narrow component from Stage II objects, but also includes a broader component from Stage 0/I objects. The YMF shape of the Ambiguous sources is a blend of these two components. (Stage III YSOs constitute only $6 \%$ of the PCYC and are not plotted.) Ambiguous YSOs dominate the (incomplete) low-mass range, which is expected because fainter sources are more likely to lack $24 \mu \mathrm{m}$ detections and other photometric measurements of sufficient quality to tightly constrain their properties. Stage 0/I YSOs are preferentially detected over Stage II YSOs at lower masses because the reddest objects are generally brighter in the mid-IR for a given mass. The steep intermediate-mass slope of the YMF traces both Stage II YSOs and Am- 

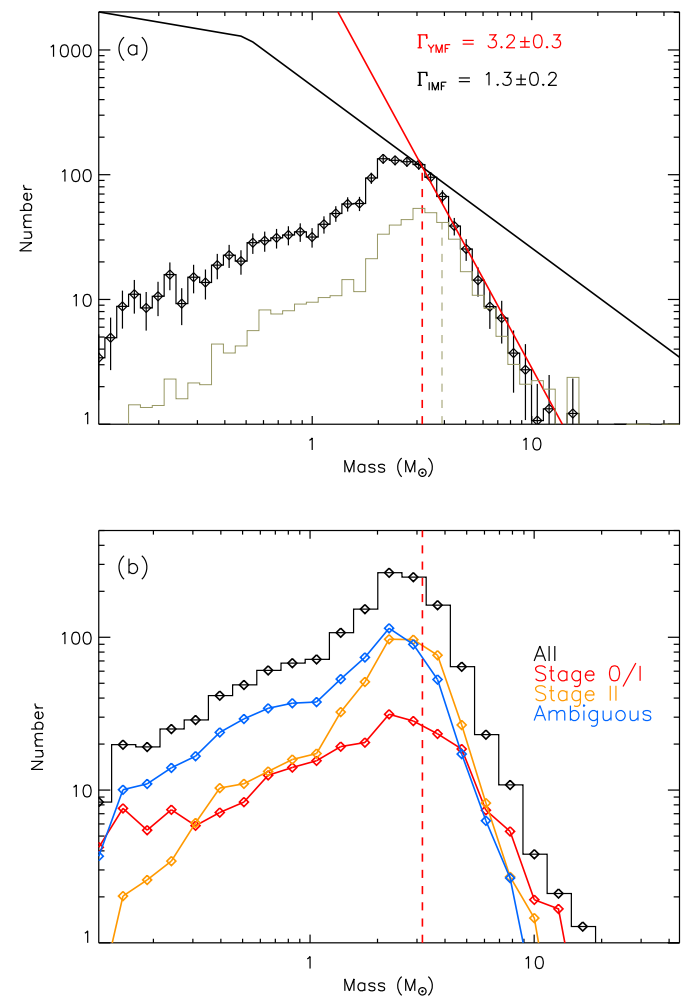

Figure 3. (a) YMF plot for all 1439 YSOs in the Catalog (histogram points with error bars). Two IMFs are overplotted: powerlaw fit to the YMF (red) over the range $m \geq M_{p}$ and Kroupa (2001) IMF scaled to match the YMF at $M_{p}=3.1 \mathrm{M}_{\odot}$ (dashed red line). The YMF from PW10 for 488 YSOs in the M17 SWex cloud is overplotted for comparison (gray histogram), and its critical mass $M_{c}=3.9 \mathrm{M}_{\odot}$ (see text) is indicated (dashed gray line). (b) Carina YMF binned up by a factor of 2 and subdivided by evolutionary stage.

biguous YSOs that are most likely dominated by Stage II objects in this mass range, given the overall distributions of Stage 0/I versus Stage II YSOs. This supports the idea that disk evolution is an important selection effect that steepens the observed YMF slope (PW10).

SP10 considered the idea that rapid disk destruction in massive stars could preferentially remove them from the YSO sample and affect the observed mass function but concluded that this effect does not significantly impact an IMF derived from the YSOs in Carina. This conclusion was based upon a perceived similarity between the empirical $K_{S}$ and [3.6] luminosity functions of the Carina YSOs and the scaled $K$ - and $L$-band luminosity functions (KLFs and LLFs) for the Orion Nebula Cluster (ONC; Muench et al. 2002). However, Muench et al. (2000) caution that LFs are not reliable tracers of IMFs when the photometry is dominated by IR excess emission. To illustrate the problem, consider the bright end of the KLF. The few most luminous YSOs in the SP10 sample, which are also included in the PCYC, have similar $M_{K}$ to $\theta^{1}$ Ori C, the most luminous star in the ONC. But while the most massive YSOs in Carina are $\sim 10 \mathrm{M}_{\odot}$ objects, $\theta^{1}$ Ori $\mathrm{C}$ is an $\mathrm{O}$ star of $\sim 40 \mathrm{M}_{\odot}$ ! While the $\mathrm{ONC}$ is a young cluster, the majority of its stars have lost their inner disks, and the KLF used by Muench et al. (2002) to derive the cluster IMF was dominated by emission from stellar photospheres. To better understand the
IMF traced by the YSOs in Carina, we rely instead on the RW06 models to relate statistically the observed IR emission to stellar mass. Our approach is subject to its own set of biases and systematics, but it has been designed for the problem at hand and has been tested in numerous other studies (e.g. Whitney et al. 2008; Povich et al. 2009; Kang et al. 2009). We are also aided by the ability to compare Carina to M17 SWex, where a similar sample of YSOs has been analyzed using the same tools (PW10).

The YMF of 488 YSOs in M17 SWex from PW10 is overplotted in Figure 3a for comparison with the PCYC. The similarity between these YMFs is striking; the only obvious difference appears to be a lower mass completeness limit for the PCYC. Both Carina and M17 are 100-pc scale GMC complexes at similar heliocentric distances, each containing several $10^{5} \mathrm{M}_{\odot}$ in molecular gas mass, and each in the process of forming a large OB association. The sensitivity of the GLIMPSE observations to YSOs in M17 SWex was limited primarily by interstellar extinction through the IR dark cloud. In contrast, the interstellar extinction to the Carina YSOs is much lower, and the sensitivity is limited instead by bright nebular emission produced by the H II regions. It is not obvious, however, why high extinction should be a much greater impediment to YSO detections than bright nebulosity. The implied difference in sensitivity is dramatic, meaning that if the YMF of PW10 is intrinsically similar to the PCYC, its completeness at $m=3.1 \mathrm{M}_{\odot}$ (corresponding to main-sequence B9 stars) is as low as $\sim 40 \%$.

The M17 SWex GMC complex appears to be forming its first generation of stars and lacks very massive, early O stars (PW10). The Carina molecular cloud complex has been thoroughly disrupted by the process of forming tens of thousands of stars, including some of the most massive stars known in the Galaxy (Smith \& Brooks 2007). The environmental differences between these two massive star-formation regions lead us to expect that there are intrinsic differences between the YSO populations, and these differences might be manifest in the YMFs. In particular, we expect that the PCYC includes a larger proportion of more evolved YSOs than the M17 SWex population.

A useful empirical comparison of the PCYC with the M17 SWex YSOs is provided by the plot of the IRAC [3.6] - [4.5] versus [4.5] - [5.8] color space presented in Figure 4. PW10 identified a class of predominantly Stage $0 /$ I YSOs that exhibited excess $4.5 \mu \mathrm{m}$ emission (denoted [4.5]E) with respect to the RW06 model SEDs and hypothesized that such sources are unresolved analogs of the extended green objects (EGOs), candidate molecular outflows from massive YSOs cataloged from the GLIMPSE images by Cyganowski et al. (2008). In this interpretation, such sources are expected to be very young, with ages $\lesssim 10^{5} \mathrm{yr}$, since outflows are powered by rapid accretion (Richer et al. 2000; Shepherd et al. 2007). We find that $\sim 50 \%$ of $[4.5] \mathrm{E}$ candidates from PW10 are found above the line

$$
[3.6]-[4.5]>1.19([4.5]-[5.8])+0.5
$$

in Figure 4; these "strong" [4.5]E sources represent $7 \%$ of the YSOs in M17 SWex, but $<1 \%$ of the PCYC. SP10 searched the Spitzer images of Carina for EGOs and found only four candidates. The corresponding dearth of 


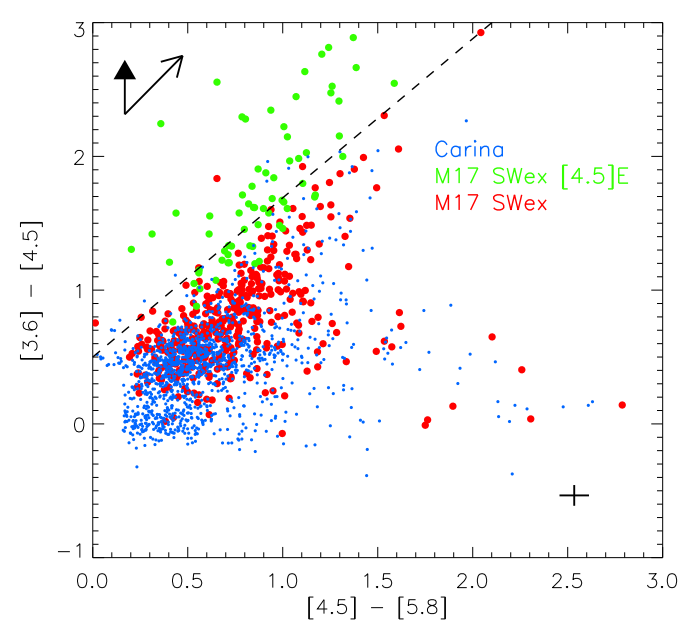

Figure 4. IRAC color space enabling the separation of YSOs with excess $4.5 \mu \mathrm{m}([4.5] \mathrm{E})$ emission. PCYC sources detected in these 3 bands are plotted along with sources from the M17 SWex YSO sample (PW10), with [4.5]E YSOs plotted in green. Candidate [4.5]E objects are located near or above the dashed line. Reddening vectors for $A_{V}=30 \mathrm{mag}$ using the extinction laws of Weingartner \& Draine (2001) and Indebetouw et al. (2005) are shown as open and filled arrows, respectively. The cross represents the typical photometric uncertainty.

[4.5]E sources supports the conclusion that these objects trace the same phenomenon as the EGOs. The difference between the two regions could be partly environmental; SP10 suggest that the harsh radiation fields permeating the Carina Nebula may dissociate molecules in the outflows, removing the source of the EGO emission. But this comparison also indicates that very young YSOs with high accretion rates are less frequent in Carina than in M17 SWex. Indeed, only $17 \%$ of the PCYC are unambiguous Stage 0/I YSOs, compared to 27\% Stage 0/I YSOs in the M17 SWex sample (PW10).

The presence of a dusty, circumstellar disk (or an envelope that will presumably evolve into a disk) is a prerequisite for inclusion in the $\mathrm{PCYC}$, hence the upper limit on the age of the YSOs is approximately the disk lifetime. The "canonical" disk lifetime for a solar-mass, classical T Tauri star is $\sim 2$ Myr (Haisch et al. 2001). If the primary mechanism for destroying dust disks around intermediate-mass YSOs is photo-evaporative dissipation driven by radiation from the central star (Hollenbach et al. 1994; Monnier \& Millan-Gabet 2002), then the disk lifetime should decrease as a strong function of stellar mass. Disk lifetimes for intermediate-mass stars, including Herbig Ae/Be stars, appear to be significantly shorter than for $\mathrm{T}$ Tauri stars, typically $\lesssim 1$ Myr (Hernández et al. 2007; Povich et al. 2009, PW10). The disk fraction among intermediate-mass stars may therefore be one of the most sensitive chronometers available for massive star-formation regions like Carina.

PW10 showed that the shape of the YMF depends critically on the mass-dependent disk fraction, and hence the recent star formation history. Assuming a constant SFR, the observed YMF slope is steepened by disk evolution above a critical mass $m>M_{c}$ for which the disk lifetime $\tau_{d}(m)$ is less than the age spread of the YSO population $\tau_{0}$. For $m \leq M_{c}, \tau_{d}(m) \geq \tau_{0}$, the disk fraction becomes unity, and the YMF traces the underlying IMF, presumably a Salpeter-Kroupa slope $\left(\Gamma_{\mathrm{YMF}} \rightarrow \Gamma_{\mathrm{IMF}}=1.3\right)$. As a result, $M_{c}$ decreases with time. Unfortunately, for the $\mathrm{PCYC} M_{c} \leq M_{p}=3.1 \mathrm{M}_{\odot}$, meaning that the critical mass falls in the range where our observations are incomplete. This limit means that the critical mass of the PCYC is lower than $M_{c}=3.9 \mathrm{M}_{\odot}$ found by PW10 for the M17 SWex sample (Figure 3a), and this could be explained by disk evolution proceeding over a longer timescale in the Carina complex. The PCYC does contain a significant population of Stage II/III YSOs with relatively evolved disks. This population is located near the line $[3.6]-[4.5]=0$ in Figure 4, hence reddening by interstellar dust is negligible, and the lack of IR excess emission at wavelengths $\leq 4.5 \mu \mathrm{m}$ suggests that the inner disks have been cleared of dust.

Assuming that the underlying IMF of the Carina young stellar population has a normal Salpeter-Kroupa form, we can estimate the present-day SFR in Carina from the PCYC. Scaling the IMF of the ONC (Muench et al. 2002) to match the PCYC YMF where it departs from a powerlaw at $M_{p}=3.1 \mathrm{M}_{\odot}$ gives a prediction of $>2 \times 10^{4}$ YSOs to a limiting mass of $0.1 \mathrm{M}_{\odot}$, corresponding to a total mass in stars of $>1.6 \times 10^{4} \mathrm{M}_{\odot}$. These numbers are lower limits due to incompleteness. Adopting a conservative upper limit of $\tau_{0} \lesssim 2 \mathrm{Myr}$ as the age spread sampled by the PCYC, we arrive at a lower limit on the recent SFR in the Carina Nebula of $>0.008 \mathrm{M}_{\odot} \mathrm{yr}^{-1}$. If the Galactic SFR is a few $\mathrm{M}_{\odot} \mathrm{yr}^{-1}$ (Murray \& Rahman 2010; Robitaille \& Whitney 2010), then the star formation activity of the entire Milky Way is equivalent to a few hundred Carina complexes.

\section{PROPERTIES OF THE X-RAY-EMITTING YSO POPULATION}

The PCYC contains $410 \mathrm{X}$-ray-detected, predominantly intermediate-mass YSOs, including 62 Stage 0/I YSOs that are candidate X-ray-emitting protostars, the largest such sample compiled to date (Table 1). Because Herbig Ae/Be stars fall into an X-ray "desert" between low-mass T Tauri stars with convection-driven emission and $\mathrm{OB}$ stars with wind-driven emission, we expect that many of the PCYC sources are intrinsically X-ray quiet. This motivates us to assume the null hypothesis that $\mathrm{X}$-ray emission apparently associated with intermediatemass YSOs is generally produced by an unresolved lowmass, T Tauri binary companion (Stelzer et al. 2006; Evans et al. 2011). In such a scenario, the intermediatemass primary is X-ray quiet but dominates the IR emission, and the physical properties derived from the IR SED modeling are expected to be uncorrelated with the presence of an X-ray-detected secondary. This null hypothesis can therefore be disproved if we observe statistically significant differences between the modeled properties of YSOs with X-ray counterparts and those without.

The detection of a faint X-ray source is highly dependent on location, because the sensitivity of the ACIS observations varies significantly across the CCCP field (Broos et al. 2011a). The sensitivity limit of the PCYC has a complicated positional dependence due to large spatial variations in extinction and nebulosity. Physical properties inferred from the IR modeling can range over orders of magnitude, and observed X-ray luminosity $L_{X}$ versus stellar mass (or $L_{X}$ versus $L_{\mathrm{bol}}$ ) relations have over an order of magnitude scatter (e.g. Preibisch et al. 2005). Potential correlations between properties derived independently from X-ray and IR observations 

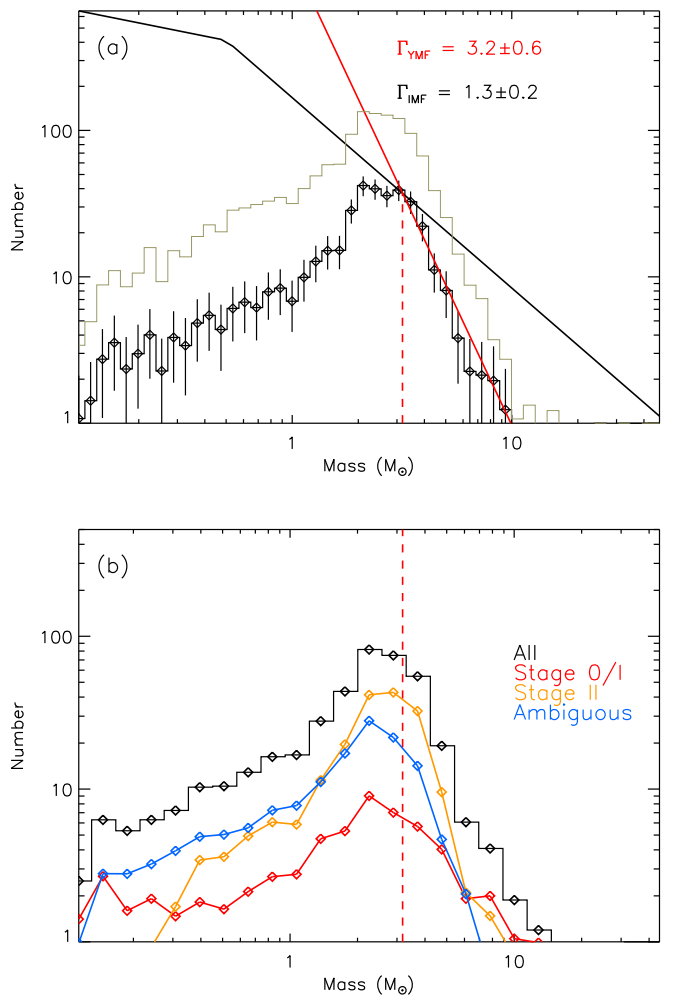

Figure 5. Same as Figure 3, except only the 410 YSOs with Xray counterparts are included in the X-YMF. The gray histogram shows the full PCYC YMF from Figure 3.

are likely to be overwhelmed by severe noise. To avoid this problem, we divide the $\mathrm{PCYC}$ into $\mathrm{CCCP}$-matched (PCYC-X) and unmatched (PCYC-U) subsamples and compare physical properties derived from SED fitting between these subsamples. The PCYC-U includes both YSOs that are intrinsically X-ray quiet and YSOs that happened to fall below the CCCP detection limit. There is likely to be substantial overlap in the physical parameters between these subsamples. Rather than attempt the potentially intractable task of correcting for the incredibly complicated, competing sensitivity variations between the X-ray and IR sample selections, we regard sample overlap as an inevitable, additional source of noise in our comparisons. At best, we might hope to discern some general, qualitative trends from the ensemble population, given the large sample size.

The YMF of the 410 YSOs in the PCYC-X (the XYMF) is plotted in Figure 5. The intermediate-mass slope $\Gamma_{\mathrm{YMF}}=3.2 \pm 0.6$ and power-law departure mass $M_{p}=3.1 \mathrm{M}_{\odot}$ (Figure $5 \mathrm{a}$ ) are consistent with the full PCYC YMF. This similarity is striking, given that we might have expected the X-YMF to skew toward lowermass YSOs drawn from the T Tauri population. Instead, there appears to be a subtle trend in the opposite direction, with the $m<2 \mathrm{M}_{\odot}$ mass range suppressed in the $\mathrm{X}-\mathrm{YMF}$. This trend is also apparent when the $\mathrm{X}-\mathrm{YMF}$ is plotted by evolutionary stage (Figure $5 \mathrm{~b}$ ).

Differences between the PCYC-X and PCYC-U are easier to discern from the cumulative mass distributions (Figure 6), constructed from the characteristic $\left\langle M_{\star}\right\rangle$ for each YSO (column 4 of Table 3 ). Two curves are plotted in each panel, representing the PCYC-U and the PCYC-
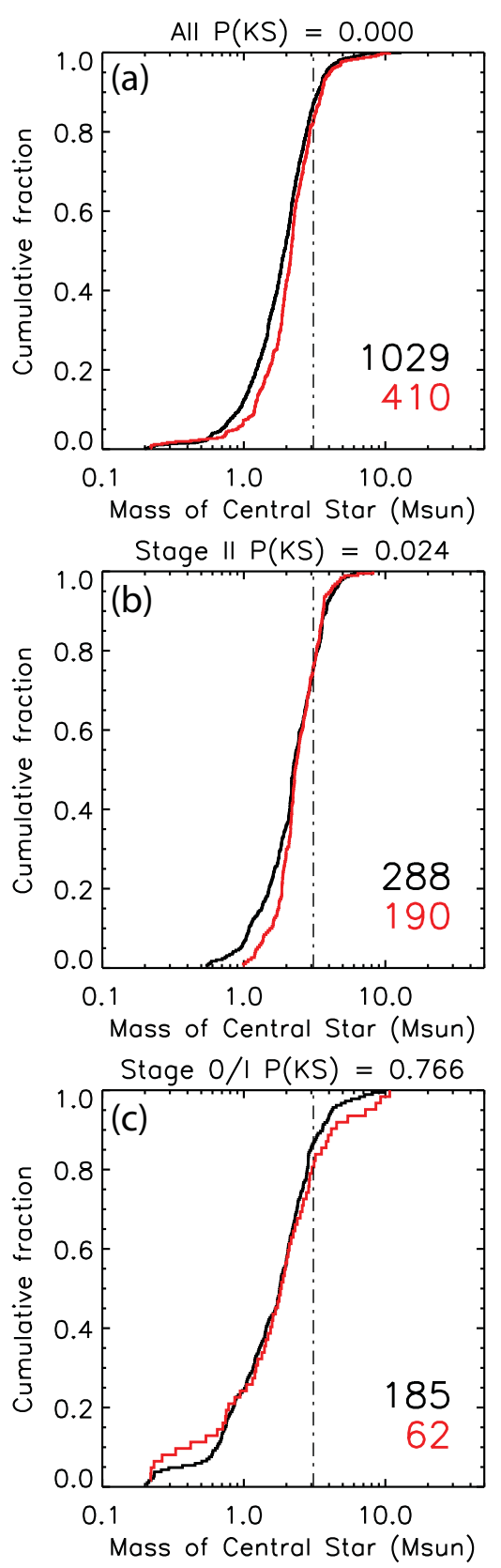

Figure 6. Cumulative mass functions comparing $\mathrm{X}$-ray detected (red) and undetected (black) YSOs: (a) All YSOs in the PCYC, (b) Stage II YSOs, and (c) Stage 0/I YSOs. The number of YSOs in each curve is printed in each panel, and each panel header gives the probability from a KS test that the two samples are drawn from the same underlying distribution. The vertical dash-dotted lines indicate the YMF power-law departure mass, $M_{p}=3.1 \mathrm{M}_{\odot}$ (see Figure 3).

$\mathrm{X}$ (red) subsamples. The panel headers give the probability $P(\mathrm{KS})$ from a Kolmogorov-Smirnov (KS) test that the two curves are drawn from the same underlying distribution. Figure $6 a$ shows that the PCYC-X as a whole includes a significantly lower fraction of sources with $m<2 \mathrm{M}_{\odot}$. Figure $6 b$ reveals that this difference is produced by the Stage II sources. Evidently the VelaCarina Spitzer observations are more sensitive than the CCCP observations to low-mass, disk-bearing stars. A KS test shows no significant difference between the cumulative mass distributions of the two samples of Stage 
0/I sources, although the two curves do appear to diverge for $m>M_{p}$ (Figure $6 c$ ).

\subsection{Intermediate-Mass, Disk-Dominated YSOs with $X$-ray Counterparts}

In Figure $6 b$ the mass distributions of both the PCYC$\mathrm{X}$ and PCYC-U samples appear to be very similar for $m \gtrsim 2 \mathrm{M}_{\odot}$. In Figure 7 we present a comparison of 6 key YSO model parameters for the unambiguous Stage II YSOs in the intermediate-mass range $1.6 \mathrm{M}_{\odot} \leq\left\langle M_{\star}\right\rangle<$ $4 \mathrm{M}_{\odot}$, equivalent to $\mathrm{A}$ through late $\mathrm{B}$ stars on the main sequence. The parameters plotted are stellar mass $\left\langle M_{\star}\right\rangle$, bolometric luminosity $\left\langle L_{\text {bol }}\right\rangle$ (which includes luminosity derived from accretion), stellar temperature $\left\langle T_{\star}\right\rangle$, normalized envelope accretion rate $\left\langle\dot{M}_{\text {env }} / M_{\star}\right\rangle$, disk mass $\left\langle M_{\text {disk }}\right\rangle$, and total extinction to the stellar surface $A_{V, t}$ (defined as the sum of the contributions to extinction produced by dust along the line of sight $A_{V}$ and circumstellar dust $\left.A_{V, c}\right)$. The characteristic values $\langle X\rangle$ of the stellar parameters $X=\left(M_{\star}, L_{\mathrm{bol}}, T_{\star}\right)$ were calculated using Equation (1), and the characteristic values of the circumstellar/environmental parameters $\langle Y\rangle$ were calculated using Equation (2).

We further subdivide our intermediate-mass Stage II sample by stellar temperature, into sets with $\left\langle T_{\star}\right\rangle<$ $5500 \mathrm{~K}$ (167 YSOs, 77 in the PCYC-X) and $\left\langle T_{\star}\right\rangle \geq$ $5500 \mathrm{~K}$ (195 YSOs, 83 in the PCYC-X). We were motivated to make this division by the hypothesis that intermediate-mass stars with cooler photospheres are more likely to have convective atmospheres and perhaps generate X-rays by a mechanism analogous to that of lower-mass T Tauri stars (e.g. Stassun et al. 2004; Preibisch et al. 2005).

We find that none of the mass, luminosity, or temperature distributions (Figures $7 a b c$ ) shows any significant correlations with the presence of X-ray emission. The normalized envelope accretion rates are low by definition for Stage II YSOs, and the distributions shown in Figure 7 confirm that these objects lack significant infalling envelopes. We do observe that the distribution functions for the low- $T_{\star}$ division (dashed curves) have systematically lower luminosity and mass compared to the high- $T_{\star}$ division, which is expected from PMS stellar evolutionary tracks.

There are no statistically significant differences in the cumulative distribution functions of the model parameters among the high- $T_{\star}$ division that correlate with Xray emission. ${ }^{17}$ This result is consistent with our null hypothesis; we cannot rule out the possibility that Xray emission associated with Stage II YSOs with $\left\langle T_{\star}\right\rangle \geq$ $5500 \mathrm{~K}$ actually originates in unresolved, lower-mass $\mathrm{T}$ Tauri companions. In contrast, the distribution functions of $M_{\text {disk }}$ and $A_{V, t}$ (Figures 7 ef) definitively fail the KS test for the low- $T_{\star}$ division and demonstrate that Xray emission is not a completely random phenomenon among the intermediate-mass Stage II YSOs. PCYC-X sources are significantly underrepresented among YSOs with the lowest disk masses, $\left\langle M_{\text {disk }}\right\rangle<10^{-3} \mathrm{M}_{\odot}($ Fig-

17 The one exception is normalized envelope accretion rate, $P(\mathrm{KS})=0.00$ in Figure $7 d$; however this is not a meaningful result because the envelope accretion rates are near zero in Stage II YSOs, and this parameter is not expected to be well constrained by the SED fitting. ure $7 e$ ). We have uncovered a population of intermediatemass PMS stars with massive disks and intrinsic X-ray emission, but the X-ray emission apparently dies off as the disks evolve. This result seems diametrically opposed to the observational paradigm in which X-ray luminosity from low-mass PMS stars increases as these objects evolve from classical T Tauri stars to weak-lined T Tauri stars (Preibisch et al. 2005; Telleschi et al. 2007), a transition that reflects the cessation of disk-fueled accretion that may suppress X-ray emission (Stassun et al. 2004).

The main result illustrated by Figure 7 is that disk mass correlates far more strongly with X-ray emission than any of the stellar parameters, demonstrating that rapid disk evolution provides a highly sensitive chronometer for intermediate-mass PMS stars. The apparent paradox with T Tauri X-ray emission is resolved when we recall that this trend is observed only among our carefully selected sub-division of intermediate-mass PMS stars with $\left\langle M_{\star}\right\rangle \geq 1.6 \mathrm{M}_{\odot}$ and $\left\langle T_{\star}\right\rangle<5,500 \mathrm{~K}$. These stars will presumably evolve into Herbig Ae/Be stars, at which point their X-ray brightness would fall below the CCCP detection limits (Stelzer et al. 2006; Broos et al. 2011a). At present, they are very young objects with relatively massive disks, still contracting toward the main sequence, and they have spectral types of G2 or later. They could thus be regarded as more massive, more luminous analogs of classical T Tauri stars.

A direct imprint of circumstellar disks on X-ray emission can be inferred from the distribution of total extinction $\left(A_{V, t} ;\right.$ Figure $\left.7 f\right)$. The top $5 \%$ of the PCYC-U reach absorptions up to $\sim 100$ times higher than the top $5 \%$ of the PCYC-X. These sources are probably highinclination systems, where nearly edge-on disks completely absorb any X-ray emission along the sightline to the central stars.

\subsection{Candidate X-ray-Emitting Protostars}

The 62 unambiguous Stage 0/I YSOs in the PCYC-X subsample are presented in Table 4, which lists values for some of the YSO model parameters along with 3 observed X-ray quantities; total-band (0.5-8 keV) net counts, median energy $\left(E_{\text {median }}\right)$, and energy flux $\left(F_{t}\right)$. As Figure $6 c$ shows, the mass distributions of Stage 0/I YSOs are very similar between the PCYC-X and PCYC-U subsamples for $\left\langle M_{\star}\right\rangle<M_{p}$. To investigate which properties other than mass may correlate with X-ray emission from protostars, in Figure 8 we present distribution functions comparing the same 6 YSO model parameters used to analyze the intermediate-mass Stage II population (Figure 7), this time plotting the unambiguous Stage 0/I YSOs with $\left\langle M_{\star}\right\rangle<2.5 \mathrm{M}_{\odot}$.

KS tests reveal that the mass distributions for these low-mass Stage 0/I YSOs are statistically the same between the PCYC-X and PCYC-U (Figure 8a), yet the luminosity distributions are significantly different, with the X-ray emitters systematically preferring lower values of $L_{\mathrm{bol}}$ (Figure $8 b$ ). Hence we may deduce that $X$-ray detected Stage 0/I YSOs are less luminous for a given stellar mass. Furthermore, both the normalized envelope accretion rates $\dot{M}_{\text {env }} / M_{\star}$ and total absorption $A_{V, t}$ (columns 6 and 7 of Table 4) show parameter distributions skewed to significantly lower values for the Xray emitters, while $T_{\star}$ is marginally higher for the $\mathrm{X}$ - 

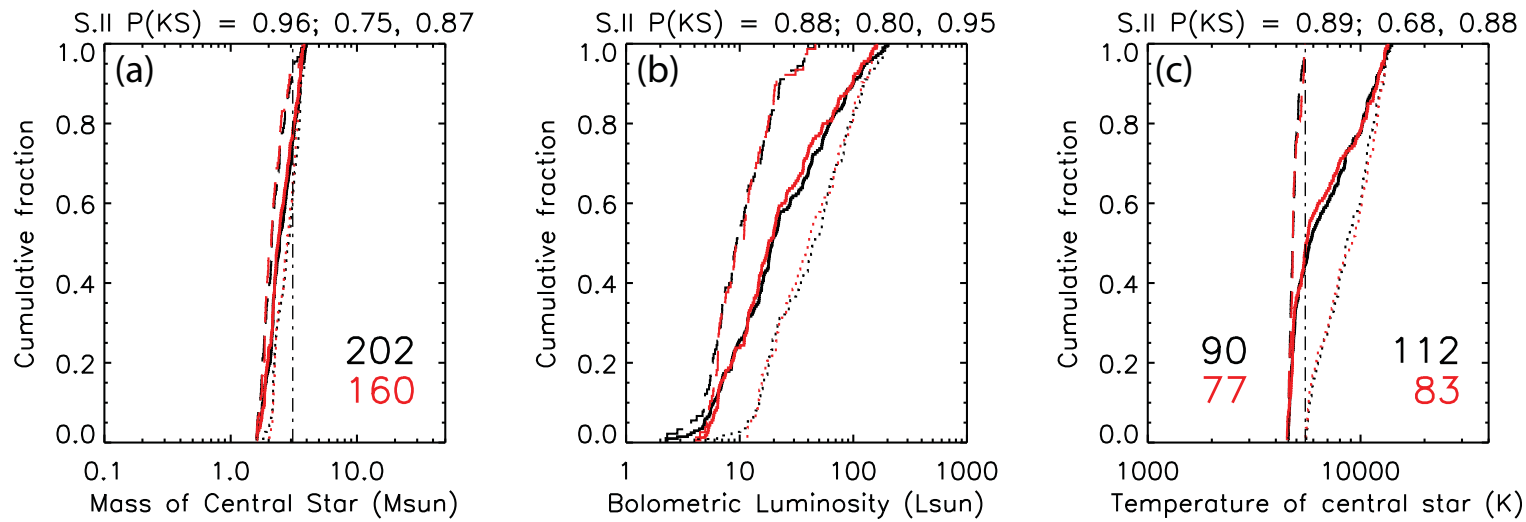

S.II $P(K S)=0.06 ; 0.86,0.00$
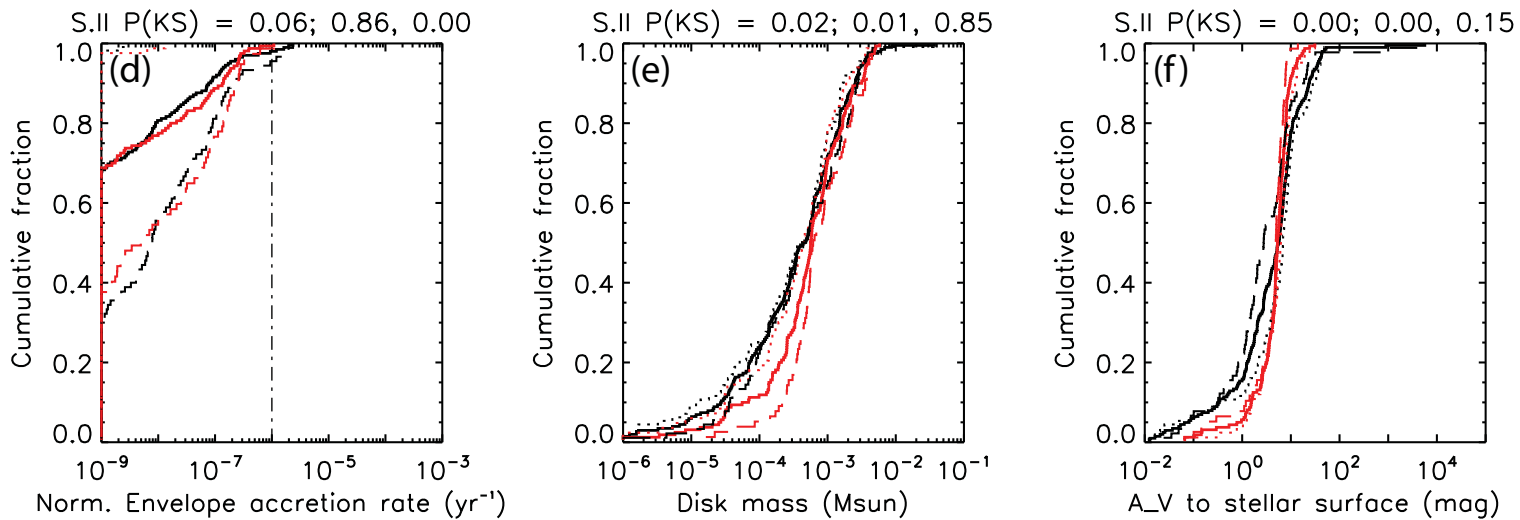

Figure 7. Cumulative distribution functions of 6 key YSO model parameters (see discussion in text) for Stage II YSOs with 1.6 M $\odot \leq$ $\left\langle M_{\star}\right\rangle<4 \mathrm{M}_{\odot}$. The dividing line at $\dot{M}_{\text {env }} / M_{\star}=10^{-6} \mathrm{yr}^{-1}$ between Stage II and Stage 0/I YSOs is the vertical dash-dotted line in panel $d$. Colors and annotations are the same as in Figure 6. The distribution functions are further subdivided by stellar temperature; long-dashed curves for $\left\langle T_{\star}\right\rangle<5500 \mathrm{~K}$ and dotted curves for $\left\langle T_{\star}\right\rangle \geq 5500 \mathrm{~K}$ (the fiducial temperature is the vertical dash-dotted line in panel $c$ ). Three KS probabilities are given in each panel header: full distribution; low- $T_{\star}$, high- $T_{\star}$ sub-distributions.
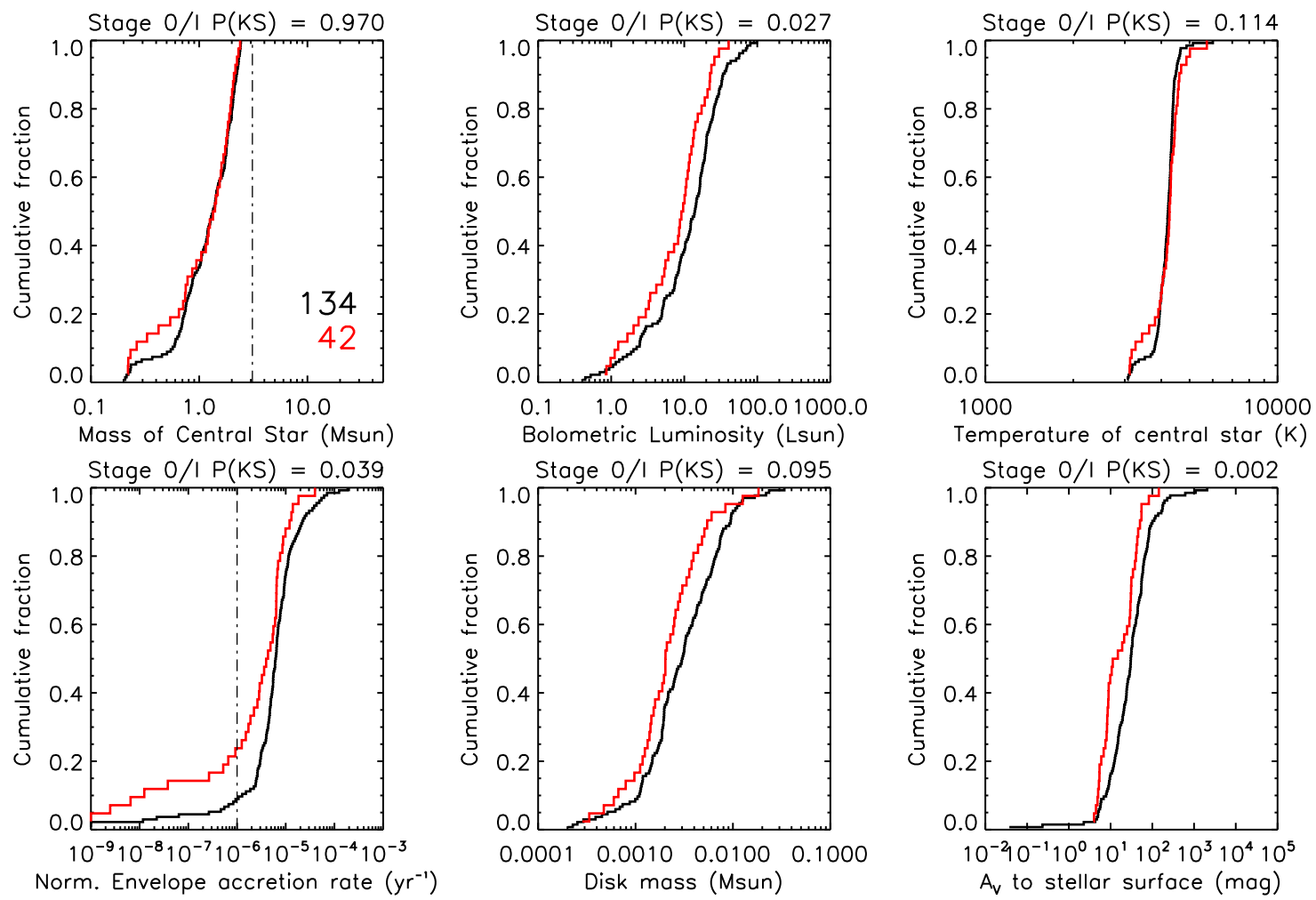

Figure 8. Cumulative distributions of 6 key YSO model parameters for Stage 0/I YSOs with $\left\langle M_{\star}\right\rangle<2.5 \mathrm{M}_{\odot}$. Colors, annotations, and fiducial lines are the same as in Figs. 6 and 7. 
ray emitters. As protostars contract, they become hotter and less luminous, their accretion rates slow, and outflow-carved, bipolar cavities grow in their envelopes, reducing the obscuration to the central star. Taken together, the distributions shown in Figure 8 demonstrate that brighter $X$-ray emission correlates with more evolved Stage 0/I YSOs.

Previous identifications of candidate X-ray emitting Class 0/I YSOs in nearby star formation regions have observed characteristically hard X-ray spectra, with $E_{\text {median }} \gtrsim 2.5 \mathrm{keV}$ (Getman et al. 2007; Prisinzano et al. 2008). X-ray spectra observed from embedded protostars are expected to be hard because soft X-rays are preferentially absorbed both by the dense circumstellar envelopes and the surrounding dense molecular cloud cores (Getman et al. 2007). About half (26/62) of our sample of candidate X-ray emitting Stage 0/I YSOs have hard Xray counterparts in the CCCP catalog $\left(E_{\text {median }}>2 \mathrm{keV}\right)$; these objects are high-probability candidate X-ray emitting protostars, grouped together in Table 4 . The remaining Stage 0/I YSOs in Table 4 are matched to soft X-ray sources, more consistent with expectations for Class II/III objects (Preibisch et al. 2005; Prisinzano et al. 2008). YSOs in this latter group may be in transition between the envelope-dominated and diskdominated phases. Many of them would not have been selected as Class 0/I objects by the conservative mid-IR empirical criteria of Prisinzano et al. (2008), for example. The model-based Stage 0/I classification, however, indicates that the YSOs in Table 4 are still very young (they possess envelopes); whether or not they are identified as "candidate protostars" is primarily a semantic choice. As expected, the IR SED modeling indicates that the soft X-ray group is less embedded than the hard X-ray group, with median $A_{V, t}=10 \mathrm{mag}$ compared to $30 \mathrm{mag}$ (column 7 of Table 4). Recalling that most YSOs in the Carina Nebula are found outside of dense molecular clouds (§4.1), the typical absorption may also be less than expected from observations of YSOs in other starforming molecular clouds that have not been disrupted by feedback from extremely massive stars.

\section{BUTTERFLY COLLECTION: INTRIGUING YSO SUB-CLUSTERS}

In this section, we identify and briefly discuss YSOs found in candidate embedded sub-clusters in the CCCP field. These YSOs are highlighted, with their group membership identified, in Tables 2 and 3 . There are a few caveats to remember. The properties of individual YSOs may not be well-constrained by the RW06 models. Sources might be multiple or otherwise confused in our multiwavelength datasets, and apparent spatial matches between CCCP sources and Vela-Carina sources do not guarantee physical association. We believe that these clusters are sufficiently interesting individual "butterflies" among the larger stellar population in the Carina complex to merit targeted follow-up multiwavelength observations that would provide higher spatial resolution, wider wavelength coverage, and/or spectroscopy.

\subsection{A Perfectly Ordinary Stage 0/I YSO Associated with an Extraordinary Obscured $X$-ray Cluster}

Townsley et al. (2011) report the discovery of a group of 4 X-ray sources in the South Pillars region that are
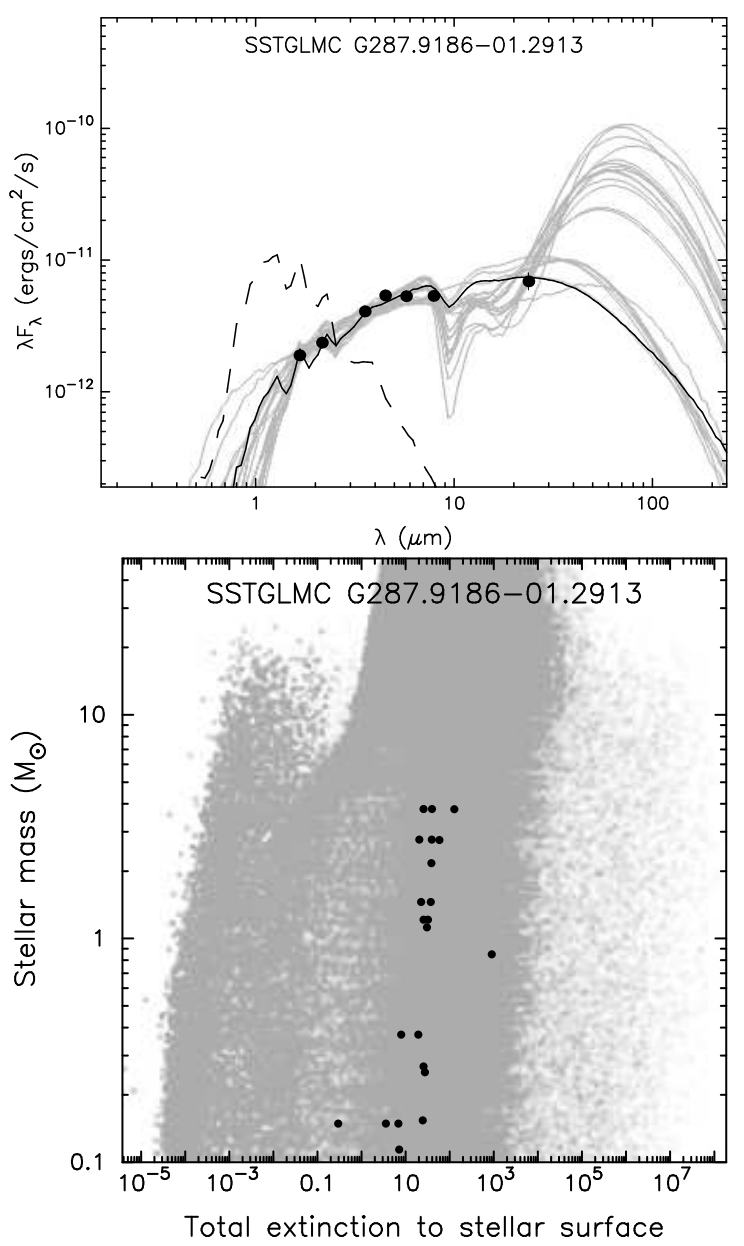

Figure 9. Top: SED of PCYC 699 (points) and well-fit YSO models (solid curves). The heavy curve represents the best-fit model, and the dashed curve is the stellar photosphere of this model as it would appear in the absence of circumstellar extinction (removing $\left.A_{V, c}\right)$. Bottom: Plot of parameters $M_{\star}$ versus $A_{V, c}$ for the set of well-fit YSO models. The gray shaded area represents the full parameter space sampled by the RW06 YSO models.

remarkably luminous, hard, and highly-obscured. Three of these sources were matched to a single Vela-Carina Catalog source, G287.9186-01.2913 (Broos et al. 2011a), or PCYC source 699 (Tables 2-4). The primary CCCP match to PCYC 699 is CXOGNC J104451.91-602511.9, the brightest $\left(F_{t}=5 \times 10^{-13} \mathrm{erg} \mathrm{cm}^{-2} \mathrm{~s}^{-1}\right)$ and secondhardest $\left(E_{\text {median }}=5.2 \mathrm{keV}\right)$ X-ray source associated with any candidate protostar in the Carina Nebula (Table 4). This source is variable and spectacularly luminous in X-rays, with $L_{X}=0.5 \mathrm{~L}_{\odot}$, corrected for $A_{V, t}=250$ mag total absorption (Townsley et al. 2011). In stark contrast, there is nothing remarkable about PCYC 699, save perhaps for the fact that its physical parameters are more poorly constrained than usual. The RW06 model fits to the SED of PCYC 699 are plotted in Figure 9 along with a parameter plot showing $M_{\star}$ versus $A_{V, c}$ for each fit. A degeneracy in the long-wavelength SED beyond $24 \mu \mathrm{m}$, where we have no data, produces 2 distinct families of model fits (Figure 9): intermediatemass YSOs with relatively high circumstellar extinction and low-mass YSOs with lower circumstellar extinction. The model fits corresponding to the highest extinction are consistent with the absorption of the X-ray source, 
but even taking into account the wide range of stellar mass and luminosity allowed by the model fits, there is no interpretation that would allow PCYC 699 to be considered a massive YSO. It is a relatively faint $24 \mu \mathrm{m}$ source, and, unlike the most luminous YSOs in the PCYC, it is neither an $M S X$ source nor an IRAS source. Thus we are left with a mystery surrounding the origin of the bright X-ray emission. The X-ray properties indicate a luminous, obscured, compact massive star cluster, but there is no commensurate IR emission.

\subsection{Five Prominent YSO Sub-Clusters or Groups}

In Figure 10 we present Spitzer multicolor images zoomed in on 5 prominent YSO clusters, 3 of them new discoveries from the combined Spitzer/Vela-Carina and CCCP observations. Extending the nomenclature of the Spitzer clusters identified by SP10, we call the new clusters Spitzer P, Q, and R (Table 3).

Spitzer $P$ and $Q:\left(\alpha_{2000}, \delta_{2000}\right)=\left(10^{h} 43^{m},-59^{\circ} 26^{\prime}\right)$. These two clusters appear in projection to be close neighbors (Figure 10a), located $\sim 8^{\prime}$ northwest of $\operatorname{Tr} 14$. Both contain a high fraction of unambiguous Stage 0/I YSOs and show no sign of dynamical relaxation, suggesting extreme youth. The location of Spitzer $\mathrm{P}$ corresponds to a $\mathrm{C}^{18} \mathrm{O}$ core (Figure 1 ), and its dense center contains a concentration of X-ray sources (Group 4; F11). Spitzer Q was not designated an X-ray group by F11, although it also coincides with an enhanced density of X-ray sources (Figure 2). Possibly the most massive Xray-emitting Stage 0/I YSO in the CCCP field is found in Spitzer Q. PCYC 179 is an extremely red Spitzer source, detected by $M S X$ but not by 2MASS (Table 2), with $\left\langle L_{\text {bol }}\right\rangle \sim 4 \times 10^{3} \mathrm{~L}_{\odot}$ and a very high envelope accretion rate, $\left\langle\dot{M}_{\text {env }}\right\rangle \sim 2.5 \times 10^{-3} \mathrm{M}_{\odot} \mathrm{yr}^{-1}$ (Table 3 ). The associated CCCP source is very hard, $E_{\text {median }}=4.0 \mathrm{keV}$ (Table 4). If the intrinsic X-ray emission is the soft spectrum produced by a normal massive stellar wind (Lucy \& White 1980), then it must be very luminous, with the soft counts almost completely absorbed. Alternatively, a more exotic emission mechanism may produce in intrinsically hard X-ray spectrum, or the CCCP source is an unresolved, low-mass companion.

Spitzer $R:\left(\alpha_{2000}, \delta_{2000}\right)=\left(10^{h} 44^{m},-59^{\circ} 08^{\prime}\right)$. In the extreme northern corner of the CCCP field we have discovered another very young, tight grouping of YSOs called Spitzer R (Figure 10b). This cluster is roughly centered on a $\mathrm{CO}$ peak that was not detected as a $\mathrm{C}^{18} \mathrm{O}$ core by Yonekura et al. (2005, and see Figure 1), and it appears to be forming intermediate-mass stars but not massive stars. There is no associated X-ray cluster, but this may simply be a sensitivity effect given that Spitzer $\mathrm{R}$ lies close to the edge of the CCCP field and was observed far off-axis by ACIS, where the sensitivity is reduced (Broos et al. 2011a).

Cr 232: $\left(\alpha_{2000}, \delta_{2000}\right)=\left(10^{h} 44.5^{m},-59^{\circ} 34^{\prime}\right)$. Cr 232 is a well-known young massive cluster to the west of $\mathrm{Tr}$ 14, containing an O3.5 V((f)) star, HD 93250, and an O5 V star, HD 303311 (Smith 2006a, and references therein). Cr 232 is a large X-ray cluster (F11) apparently centered near the end of a broad dust pillar oriented toward $\eta$ Car and Tr 16 (Figure 10c). Cr 232 is undoubtedly richer than the newly-discovered Spitzer YSO clusters. Its location suggests that its formation may have been triggered recently by feedback from the massive stars in Tr 16. Apparently in the middle of the dust pillar associated with $\mathrm{Cr} 232$ we detect a candidate Xray-emitting, highly-embedded, massive Stage 0/I YSO, PCYC 556 (Table 3), similar to PCYC 179 in Spitzer $\mathrm{Q}$, but the associated X-ray source is fainter by 0.4 dex and has $E_{\text {median }}=1.9 \mathrm{keV}$, placing it among the soft $\mathrm{X}$-ray group in Table 4. PCYC 556 is among the reddest IRAC sources in the PCYC ([3.6] - [4.5] = $1.4 \mathrm{mag}$, $[5.8]-[8.0]=1.0 \mathrm{mag}$; Table 2$)$. This relatively soft Xray emission apparently originating from an embedded massive star suggests a highly luminous, intrinsically soft $\mathrm{X}$-ray spectrum, perhaps powered by shocks in a strong stellar wind or accretion flow. Alternatively, the CCCP source may actually be a less obscured star that happens to be confused with the bright IRAC source.

Spitzer O: $\left(\alpha_{2000}, \delta_{2000}\right)=\left(10^{h} 45^{m},-60^{\circ} 26.5^{\prime}\right)$. Identified recently by SP10, Spitzer O, located in the extreme southeast corner of the South Pillars, is a far outpost of massive star formation in the Carina complex (Figure $10 d)$. The cluster is associated with the southern of two $\mathrm{C}^{18} \mathrm{O}$ cores in the molecular cloud known as the Giant Pillar (Figure 1). Although it is near the edge of the CCCP field, where the ACIS sensitivity is lower, Spitzer O is nevertheless associated with a surface density enhancement in the X-ray sources (Figure 2), which was not listed by F11 on account of its remote location. Spitzer O includes a small IR dark cloud that hosts one of the few examples of an EGO found in the Carina Nebula (SP10). This EGO appears to be associated with 4 Stage 0/I YSOs (Table 3 ) in a tight, linear grouping. Extending this line westward along the IR dark cloud (to the right in Figure $10 d$ ), we find a Stage II YSO and a candidate low-luminosity ultracompact H II region, suggesting a time-sequence of intermediate-mass star formation. Further to the west, Spitzer O also contains at least one massive star inside a compact $\mathrm{H}$ II region, identifiable as a small bubble outlined in $8 \mu \mathrm{m}$ emission containing diffuse $24 \mu \mathrm{m}$ emission from dust heated by the central ionizing source, most likely a late O-type star (P11). Two similar but slightly less bright H II regions, complete with their own ionizing stars, are visible to the north and south of cluster $\mathrm{O}$. All 3 candidate $\mathrm{OB}$ stars in Figure $10 d$ are detected in X-rays, and none appears to have been previously cataloged (P11).

\section{DISCUSSION}

\subsection{A Recent Star Formation History for the Carina Nebula}

In $\S 4.2$, we derived a lower limit on the recent, global SFR in the Carina Nebula of $>0.008 \mathrm{M}_{\odot} \mathrm{yr}^{-1}$, averaged over the past $\tau_{0} \lesssim 2 \mathrm{Myr}$, traced by the IR excess sources included in the PCYC. Feedback from massive stellar winds and radiation has been invoked by many authors as the driver of ongoing star formation (e.g., Smith et al. 2000; Smith \& Brooks 2007, SP10). If triggering by massive stars is indeed the dominant mode of continuing star formation in GMCs that host energetic H II regions, we can assess its relative importance by comparing the present-day SFR to the historical SFR that produced the massive star clusters that ionize the Carina Nebula and provide the source of the feedback. 

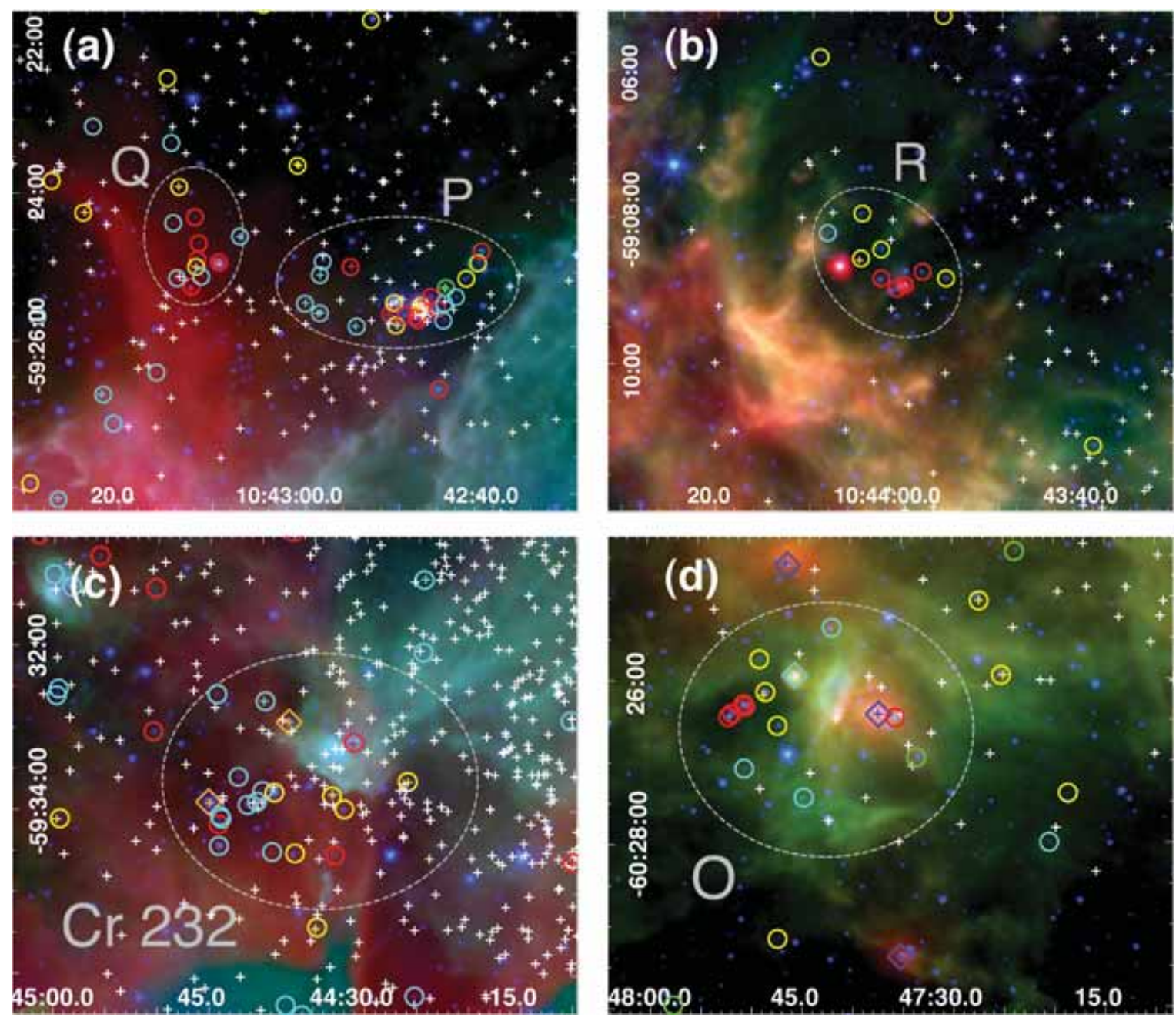

Figure 10. Spitzer color images (red $=24 \mu \mathrm{m}$, green $=8.0 \mu \mathrm{m}$, blue $=4.5 \mu \mathrm{m}$ ) highlighting the 5 tight groupings or clusters of YSOs discussed in the text. In all panels, YSOs are marked by circles color-coded by most probable evolutionary stage as in Fig. 1, and crosses mark the positions of CCCP X-ray catalog sources. Where an X-ray source has been matched to a YSO, the crosses have been colored to match the YSO classification. Dashed ovals have been drawn subjectively to enclose the YSOs that appear associated with each grouping. Coordinate axes are equatorial (J2000). In panel (c), two cataloged, X-ray-emitting early O stars in Cr 232 are marked by orange diamonds and crosses; these are HD 93250 (left) and HD 303311 (right). Regions at the bottom edge of this image appear turquoise due to saturation of the $24 \mu \mathrm{m}$ nebular emission. In panel (d), three candidate X-ray detected OB stars from P11 are marked by blue diamonds and crosses, and a candidate ultracompact $\mathrm{H}$ II region is highlighted with a cyan diamond.

Smith \& Brooks (2007) estimated a total stellar population of $5-8 \times 10^{4}$ stars down to the hydrogen-burning limit by extrapolating from the known O-type stars in Carina (Smith 2006a). The relative rarity of O stars requires an enormous extrapolation over the IMF to reach the low-mass stars that contain the bulk of the mass (Kroupa 2001). Measurements of the IMF among low-mass stellar populations (from the Galactic field and open clusters) and high-mass stellar populations (from very massive Galactic and extragalactic clusters) have generally found slopes consistent with the SalpeterKroupa value, but region-to-region variations do exist (Bastian et al. 2010). Because very massive clusters with a well-populated upper IMF are located at such large distances that individual low-mass stars generally cannot be resolved, there has been no simultaneous, self-consistent measurement of the IMF from solar-mass stars to the most massive stars. Compounding the problem, theoretical calibrations of the masses of $\mathrm{O}$ stars encounter a factor of $\sim 2$ discrepancy between "spectroscopic" masses derived from surface gravity and "evolutionary" masses expected from stellar structure (Martins et al. 2005; Weidner \& Vink 2010). Finally, in the specific case of the Carina Nebula the completeness of the sample of known O stars (Smith 2006a) may be as low as 50\% (P11).

The CCCP X-ray catalog itself offers an independent measure of the total stellar population. The X-ray luminosity function (XLF) has proven to be a reliable tracer of the IMF (Getman et al. 2006) that is most sensitive to low- and intermediate-mass PMS stars. F11 scale the XLF from the Chandra Orion Ultradeep Project (COUP; Feigelson et al. 2005), for which the underlying IMF is well-characterized (Muench et al. 2002), to the XLFs from 4 distinct broad spatial regions within the CCCP and predict a total population of $\sim 10^{5}$ stars above the brown dwarf mass limit. Because X-ray detection efficiencies are lower for YSOs versus diskless PMS stars (Preibisch et al. 2005) and for heavily obscured versus lightly obscured stars (e.g. Figure 3 in Feigelson et al. 2005), the simple XLF scaling analysis implicitly assumes that any differences in the global disk fractions or the 


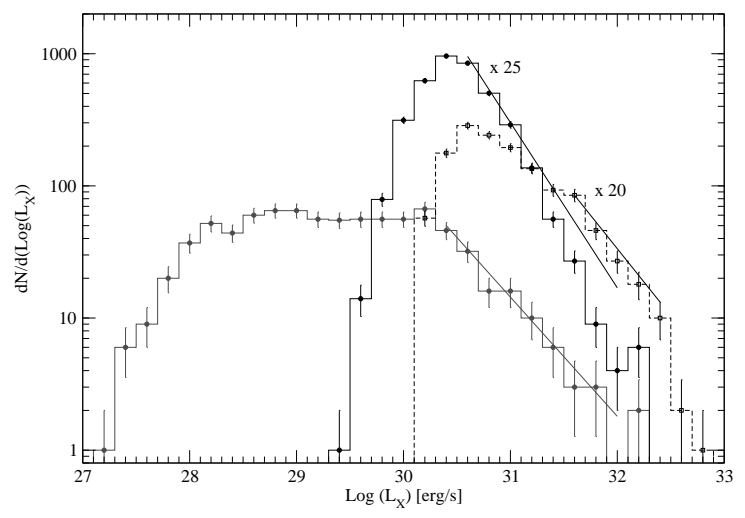

Figure 11. Comparison of global total-band (0.5-8 keV), absorption-corrected X-ray luminosity functions (XLFs) for diskless candidate PMS stars in the CCCP to the XLF (gray histogram) of 839 lightly obscured cool stars from the COUP sample (Feigelson et al. 2005). XLFs are analyzed separately for the lightly-obscured ( $E_{\text {median }} \lesssim 2 \mathrm{keV}$; solid black histogram) and heavily-obscured $\left(E_{\text {median }}>2 \mathrm{keV}\right.$; dashed histogram) CCCP samples, and the scale factors used to match the COUP XLF to each CCCP XLF are labeled on the plot. Best-fit (negative) power-law slopes to each XLF are overplotted as lines; these are $\Gamma_{\mathrm{XLF}}=0.9,1.3$, and 1.0 for the COUP, lightly-obscured CCCP, and heavily-obscured CCCP XLFs, respectively.

distributions of X-ray absorption between COUP and CCCP are negligible.

In Figure 11 we present a more detailed analysis of the CCCP XLF. Two XLFs, separated according to median energy into lightly-obscured $\left(E_{\text {median }} \leq 2 \mathrm{keV}\right)$ and heavily-obscured $\left(E_{\text {median }}>2 \mathrm{keV}\right)$ samples, were constructed using all CCCP sources identified as cluster members that were sufficiently bright to enable the derivation of absorption-corrected luminosities $L_{X}$ (Getman et al. 2010; Broos et al. 2011b). The 410 CCCP sources matched to YSOs in the PCYC were excluded, as were $\sim 200 \mathrm{X}$-ray detected known or candidate OB stars (P11). The resulting XLFs (Figure 11) are clean samples of diskless PMS stars that can be more directly compared to the 839 lightly obscured stars in the COUP $\mathrm{XLF}$, the vast majority of which lack circumstellar disks (Feigelson et al. 2005). The scale factors between the COUP and CCCP XLFs are 25 and 20, for the lightly and heavily obscured samples, respectively, giving a total of $45 \times 839=3.8 \times 10^{4}$ diskless CCCP cluster members with masses $>0.1 \mathrm{M}_{\odot}$. This result is a lower limit due to the assumption that sources for which $L_{X}$ could not be obtained actually have $L_{X}$ values below the XLF turnoffs at $\log \left(L_{X} /\left[\mathrm{erg} \mathrm{s}^{-1}\right]\right) \sim 30.6$ and 31.6 for the lightly and heavily obscured samples, respectively (Figure 11).

Combining $\gtrsim 3.8 \times 10^{4}$ diskless stars traced by the XLF (Figure 11) with $\gtrsim 2 \times 10^{4}$ disk-bearing YSOs predicted by the YMF (Figure 3 ) gives $\gtrsim 5.8 \times 10^{4}$ young stars for the global Carina Nebula population, in agreement with the estimates of F11 and Smith \& Brooks (2007). The total stellar mass is $4.9-8.6 \times 10^{4} \mathrm{M}_{\odot}$ (the upper bound is computed using the XLF scaling analysis from F11). Because the large majority of CCCP sources classified as likely Carina members (Broos et al. 2011b) are associated with the clustered stellar population (F11), we deduce that the age spread represented by the global XLF is similar to the $\sim 5$ Myr age spreads measured for the major ionizing clusters (Wang et al. 2011; Wolk et al. 2011, and references therein). The time-averaged global SFR has been $0.010-0.017 \mathrm{M}_{\odot} \mathrm{yr}^{-1}$ over the past $\sim 5 \mathrm{Myr}$.

The combined YMF and XLF results above lead straightforwardly to a global mid-IR excess fraction of $\sim 30 \%$ among the Carina Nebula population. Compared to the commonly-used $K_{S}$-excess fraction, this mid-IR excess fraction is much closer to the astrophysical fraction of stars with inner disks. YSOs dominated by circumstellar disks or envelopes do not separate cleanly from reddened, diskless stars in $J H K_{S}$ color-color diagrams (Whitney et al. 2003, RW06). Applying the $K_{S}$-excess selection criteria of Preibisch et al. (2011) to the PCYC sources detected in 2MASS $J H K_{S}$ (Table 2), we find that only $36 \%$ of mid-IR selected YSOs exhibit a $K_{S}$ excess. We thus predict a global $K_{S}$ excess fraction among the Carina Nebula population of $30 \% \times 36 \%=11 \%$. This result agrees very well with the $\lesssim 10 \%$ global $K_{S}$-excess fraction reported by Preibisch et al. (2011) from deep $J H K_{S}$ imaging of the CCCP stellar population, considering that the X-ray detection frequency is expected to be lower for YSOs than for diskless PMS stars. We note that the stellar population in the Carina Nebula is far from coeval, hence large variations from the $\sim 30 \%$ global average mid-IR excess fraction are expected among different sub-populations, for example the relatively old Tr 15 cluster (Feinstein et al. 1980 ) has a very low disk fraction (see Figure 2 and also Wang et al. 2011) while the very young Treasure Chest cluster may have a disk fraction near 100\% (Smith et al. 2005). The disk fraction also decreases as a function of increasing stellar mass (PW10); the global value is dominated by the numerous low-mass stars, while the $\mathrm{OB}$ stellar population generally exhibits a low frequency of marginal mid-IR excess emission that likely is not indicative of circumstellar disks (P11).

In the South Pillars region, SP10 observed a general pattern of YSOs distributed spatially in the cavities between the ends of pillars and the ionizing stars and concluded that when star formation is triggered by feedback from massive stars, YSOs are left in the wake of retreating, evaporating pillars and molecular cloud surfaces eroded by advancing ionization fronts. Just over $50 \%$ of the YSOs in the PCYC are located in the South Pillars field presented by SP10 (Figures 1 and 2). The wide-field coverage of the Vela-Carina survey allows us to generalize this picture of ongoing, potentially feedback-driven star formation beyond the South Pillars to the entire Carina Nebula. If triggering were indeed the dominant mode for ongoing star formation, then the high presentday SFR in the Carina Nebula would demonstrate that feedback can provide the mechanism for sustaining and propagating star formation throughout a GMC complex.

We caution, however, that while most of the active star formation has migrated outward (Smith \& Brooks 2007), making a clear division between a triggered YSO population and the OB stars that provide the trigger would be an oversimplification. The large concentrations of YSOs associated with the central, massive $\operatorname{Tr} 16$ and $\operatorname{Tr} 14$ clusters (Figures 1 and 2) indicate that these clusters are hotbeds of recent star formation; they are still being built. In contrast, the older Tr 15 and Bochum 11 clus- 
ters, both located in the outer regions of the Carina Nebula, have no associated YSO sub-clusters even though both are obvious X-ray star clusters (Figure 2). The Carina Nebula is famous for its evolved super-massive stellar population, $\eta$ Car being the capstone, but the numerous OB stars apparently associated with compact clusters or groups of YSOs (Figure 10, and see also Figure 5 of P11) could be as young as a few $10^{5} \mathrm{yr}$, especially considering that the intense winds and radiation fields of massive stars likely destroy their natal envelopes over very short timescales compared to the disk lifetimes of lower-mass stars (Hollenbach et al. 1994).

The triggered star formation hypothesis is very difficult to test in a region as complicated as Carina; proof would require additional information beyond the observed correlation between YSOs and ionization fronts to establish a causal relationship that the ongoing star formation could not have occurred in the absence of a triggering mechanism provided by the massive stars. Highresolution interferometric radio or sub-mm detections of dense, massive, molecular cores that lack active star formation and remain protected from external radiation fields within the Carina molecular clouds could provide such evidence.

An alternate hypothesis of turbulence-driven, hierarchical fragmentation and collapse of the Carina GMC complex (Sabbi et al. 2007, and references therein) also appears consistent with currently available data. This idea could explain the cluster-of-clusters morphology of the Carina stellar populations and is supported by our results that a large, approximately coeval YSO population is widely distributed throughout the Carina complex and the present-day SFR is approximately the same as the time-averaged historical SFR. In this scenario, feedback from massive stars need not trigger star formation, but it could regulate star formation by rapidly sweeping the natal molecular cloud fragments away from young embedded clusters, revealing and even unbinding these YSO clusters (as suggested by SP10) over shorter timescales than expected from observations of more pristine molecular cloud environments such as M17 SWex (PW10).

In summary, the global star formation history of the Carina GMC complex is characterized by an approximately constant SFR of $0.010-0.017 \mathrm{M}_{\odot} \mathrm{yr}^{-1}$ over the past $\gtrsim 5 \mathrm{Myr}$, perhaps set in motion by the hierarchical collapse and fragmentation of the GMC into a cluster of clusters. This average SFR has been punctuated by intense bursts of massive star formation. The largest burst produced the super-massive stellar population in Tr 16, including $\eta$ Car, about 3-4 Myr ago (e.g. DeGioiaEastwood et al. 2001; Wolk et al. 2011), followed by a second burst that formed the very massive stellar population in the core of the $\operatorname{Tr} 14$ cluster, perhaps as recently as 0.5 Myr ago (Sana et al. 2010). Subsequent to the formation of the very massive ionizing clusters, the recent star formation history in the Carina Nebula has been driven or at least regulated by feedback from the massive stars.

\subsection{The Production of X-rays by Intermediate-Mass PMS Stars}

Our comparative study in $\S 5$ between the subpopulations of the PCYC with and without X-ray matches in the CCCP catalog (Broos et al. 2011a) reveals some qualitative trends that provide clues into the origin of Xray emission in intermediate-mass PMS stars. Across the $\lesssim 1-10 \mathrm{M}_{\odot}$ mass range sampled by the PCYC (Figure 5), $\widetilde{\mathrm{X}}$-ray emission appears to be activated during the protostellar evolutionary phase, and this emission becomes more readily detectable in relatively evolved Stage 0/I objects for which the absorption to the central star is reduced. It is well-established that the X-ray luminosity of low-mass, T Tauri stars increases as they evolve from disk-dominated (Stage/Class II) to diskless (Class III) PMS stars (Stassun et al. 2004; Telleschi et al. 2007; Prisinzano et al. 2008). In stark contrast, we find that intermediate-mass, disk-bearing YSOs continue to produce X-rays as they evolve beyond the Stage 0/I phase, but this emission fades away as the circumstellar disks evolve and the central stars pass through a Herbig Ae/Be phase during their approach to the main sequence.

$\mathrm{X}$-ray detections of intermediate-mass, disk-dominated YSOs in the mass range $1.6-4 \mathrm{M}_{\odot}$ are correlated with higher disk mass only for stars with cool photospheric temperatures (Figure 7). Although these stars will arrive on the main sequence as $\mathrm{A}$ and $\mathrm{B}$ stars with $T_{\text {eff }} \geq$ $7500 \mathrm{~K}$ and fully radiative envelopes, currently they have $T_{\text {eff }}<5500 \mathrm{~K}$ and spectral types later than G2. During their early PMS evolution, intermediate-mass stars can thus be regarded, in terms of their stellar structure and surface temperatures, as more luminous analogs of classical T Tauri stars, complete with convective envelopes. We therefore suggest that X-ray emission from intermediate-mass YSOs originates in a scaled-up version of the mechanism powering X-rays from $\mathrm{T}$ Tauri stars; convection-driven magnetic reconnection activity (Stassun et al. 2004; Preibisch et al. 2005). Because intermediate-mass stars reach the main sequence within a few Myr (Siess et al. 2000), this emission mechanism is available for only a short time compared to low-mass PMS stars. The rapid fading of X-ray emission coincides with the rapid dissipation of the circumstellar disk as the temperature of the central star rises.

This rapidly fading dynamo hypothesis can also explain a puzzling feature of the CCCP XLF. The slope of the lightly-obscured CCCP XLF, fitted with a powerlaw function using the method of Maschberger \& Kroupa (2009), is $\Gamma_{\mathrm{XLF}}=1.3$, significantly steeper than the slopes of both the the comparison lightly-obscured COUP XLF $\left(\Gamma_{\mathrm{XLF}}=0.9\right)$ and the heavily-obscured CCCP XLF $\left(\Gamma_{\mathrm{XLF}}=1.0\right.$; Figure 11$)$. If the lightlyobscured CCCP XLF traces an older stellar population compared to either the COUP or the heavilyobscured CCCP XLFs, then a relatively rapid decay in $L_{X}$ among the more luminous, intermediate-mass stars would steepen the observed XLF slope, just as massdependent circumstellar disk evolution steepens the observed YMF slope (Figure 3). A similar deficit of stars with $\log L_{X} \gtrsim 31 \mathrm{erg} \mathrm{s}^{-1}$ steepens the observed XLF slope of the Tr 15 cluster (Wang et al. 2011). With an age $\gtrsim 5 \mathrm{Myr}$, Tr 15 represents a significantly older stellar population compared to either the $\mathrm{ONC}$ or the other massive clusters in the Carina Nebula.

\section{CONCLUSIONS}

We have used the Spitzer Vela-Carina point-source lists, in combination with complementary IR datasets, to carry out an unbiased search for YSOs with IR excess 
emission throughout the Carina Nebula. We produce a Pan-Carina YSO Catalog (PCYC) of 1439 highly-reliable candidate YSOs, 410 of which have X-ray counterparts from the CCCP catalog. The PCYC comprises one of the largest samples of intermediate-mass YSOs in a single massive star-forming complex compiled to date, and it is the largest such collection with a complete set of complementary X-ray observations. From our initial analysis of this rich dataset, we can draw the following conclusions:

- The mass function (YMF) of the Carina YSOs exhibits a power-law slope $\Gamma_{\mathrm{YMF}}=3.3$, significantly steeper than the Salpeter-Kroupa IMF. Such a steep slope, first observed for the YSO population of the M17 SWex IR dark cloud, is at least partially the result of an observational selection effect caused by rapid disk evolution among intermediate-mass YSOs (PW10). M17 SWex and Carina are very different environments; the former is a cold, IR dark cloud apparently experiencing its first wave of star formation while the latter is an evolved $\mathrm{H}$ II region powered by some of the most massive stars in the Galaxy. While the YMF slopes are similar, the lower critical mass of the PCYC sample, below which the YMF may agree with a normal Salpeter-Kroupa IMF, likely reflects a larger age spread among the YSOs in the Carina Nebula.

- The time-averaged global SFR in the Carina complex has been $0.010-0.017 \mathrm{Msun}_{\mathrm{yr}}{ }^{-1}$ over the past $<5 \mathrm{Myr}$, punctuated by intense bursts of activity that produced the very massive stellar populations in the $\operatorname{Tr} 16$ and $\operatorname{Tr} 14$ clusters. If the presentday Galactic SFR is a few $\mathrm{M}_{\odot} \mathrm{yr}^{-1}$, then the star formation activity of the entire Milky Way is equivalent to a few hundred Carina complexes. The cluster-of-clusters pattern of star formation in the Carina Nebula is consistent with hierarchical fragmentation and collapse of a GMC complex (Sabbi et al. 2007).

- The present-day global star formation rate in the Carina Complex is $>0.008 \mathrm{M}_{\odot} \mathrm{yr}^{-1}$. The massive central ionizing clusters Tr 16 and Tr 14 still host intense concentrations of ongoing star formation. Ongoing star formation activity is distributed widely throughout Carina, driven or regulated by feedback from the massive stellar population, which acts to quickly reveal and even unbind young, embedded clusters (SP10).

- The PCYC includes 62 candidate X-ray-emitting Stage 0/I YSOs, a sample large enough to provide statistical evidence that X-ray detections correlate with more evolved protostars. Our results support previous findings that the physical mechanism producing X-rays in PMS stars is activated during the protostellar evolutionary phase, and circumstellar material contributes significantly to the absorption of X-rays from the youngest YSOs (Getman et al. 2007; Prisinzano et al. 2008).

- The apparent X-ray emission from intermediatemass YSOs is usually consistent with the presence of unresolved, low-mass companions. But we also isolate a class of intermediate-mass Stage II YSOs with cooler photospheres and relatively massive circumstellar disks for which the X-ray emission appears to be intrinsic. We suggest that the progenitors of Herbig Ae/Be stars produce X-rays during their early PMS evolution, perhaps powered by magnetic reconnection activity during a convective atmosphere phase, but this mechanism decays as the stars approach the main sequence. A rapid fading of the stellar dynamo could thus act to steepen observed XLF slopes as a function of increasing cluster age (Wang et al. 2011).

- We identify 3 new very young, tight YSO subclusters in the northwestern region of the Carina Nebula, 2 of which are associated with significant concentrations of X-ray sources (F11).

The full PCYC dataset is available in the electronic versions of Tables 2 and 3 . We hope that this catalog will serve as a valuable database for future multiwavelength observations and modeling of star formation in the Carina Complex.

We thank E. D. Feigelson, T. Preibisch, and T. Montmerle for insightful conversations that helped improve this work. We thank the anonymous referee for a timely and valuable review. M.S.P. is supported by an NSF Astronomy and Astrophysics Postdoctoral Fellowship under award AST-0901646. This work is based on observations from the Spitzer Space Telescope GO programs 30848 (MIPSCAR; PI: N.S.) and 40791 (Vela-Carina; PI: S.R.M.). This publication makes use of data products from the Two Micron All-Sky Survey, which is a joint project of the University of Massachusetts and the Infrared Processing and Analysis Center/California Institute of Technology, funded by NASA and the NSF. Support for this work was provided by NASA through awards issued by the Jet Propulsion Laboratory, California Institute of Technology. This work was also supported by Chandra X-ray Observatory grant GO-9131X (PI: L.K.T.) and by the ACIS Instrument Team contract SV4-74018 (PI: G. Garmire), issued by the Chandra Xray Center, which is operated by the Smithsonian Astrophysical Observatory for and on behalf of NASA under contract NAS8-03060. K.G.S. acknowledges support from NSF grant AST-0849736.

Facilities: $\quad$ Spitzer(IRAC), Spitzer(MIPS), CTIO:2MASS, MSX, CXO (ACIS)

\section{REFERENCES}

Ascenso, J., Alves, J., Vicente, S., \& Lago, M. T. V. T. 2007, A\&A, 476, 199

Bastian, N., Covey, K. R., \& Meyer, M. R. 2010, ARA\&A, 48, 339 Benjamin, R.A. et al. 2003, PASP, 115, 953

Broos, P. S. et al. 2011a, ApJS, in press (CCCP Catalog Paper)

Broos, P. S. et al. 2011b, ApJS, in press (CCCP Classifier Paper) Carey, S. J. et al. 2009, PASP, 121, 76

Castelli, F., \& Kurucz, R. L. 2004, arXiv:astro-ph/0405087

Cyganowski, C. J. et al. 2008, AJ, 136, 2391

DeGioia-Eastwood, K., Throop, H., Walker, G., \& Cudworth, K. M. 2001, ApJ, 549, 578

Evans, N. R. 2011, ApJS, in press (CCCP Tr16 B Stars Paper)

Fazio, G. G. et al. 2004, ApJS, 154, 10

Flaherty, K. M. et al. 2007, ApJ, 663, 1069 
Feigelson, E. D., et al. 2005, ApJS, 160, 379

Feigelson, E. D. et al. 2011, ApJS, in press (CCCP clustering paper; F11)

Feinstein, A., Moffat, A. F. J., \& Fitzgerald, M. P. 1980, AJ, 85, 708

Feldmeier, A., Puls, J., \& Pauldrach, A. W. A. 1997, A\&A, 322, 878

Garmire, G. P., Bautz, M. W., Ford, P. G., Nousek, J. A., \& Ricker, G. R., Jr. 2003, SPIE, 4851, 28

Grabelsky, D. A., Cohen, R. S., Bronfman, L., \& Thaddeus, P. 1988, ApJ, 331, 181

Getman, K. V., Feigelson, E. D., Townsley, L., Broos, P., Garmire, G., \& Tsujimoto, M. 2006, ApJS, 163, 306

Getman, K. V., Feigelson, E. D., Garmire, G., Broos, P., \& Wang, J. 2007, ApJ, 654, 316

Getman, K. V., Feigelson, E. D., Broos, P. S., Townsley, L. K., \& Garmire, G. P. 2010, ApJ, 708, 1760

Haisch, K. E., Lada, E. A., \& Lada, C. J. 2001, AJ, 121, 2065

Hernández, J. et al. 2007, ApJ, 662, 1067

Hollenbach, D., Johnstone, D., Lizano, S., \& Shu, F. 1994, ApJ, 428,654

Indebetouw, R. et al. 2005, ApJ, 619, 931

Indebetouw, R. et al. 2007, ApJ, 666, 321

Kang, M., Bieging, J. H., Povich, M. S., \& Lee, Y. 2009, ApJ, 706,83

Koenig, X. P., Allen, L. E., Gutermuth, R. A., Hora, J. L., Brunt, C. M., \& Muzerolle, J. 2008, ApJ, 688, 1142

Kroupa, P. 2001, MNRAS, 322, 231

Lucy, L. B., \& White, R. L. 1980, ApJ, 241, 300

Martins, F., Schaerer, D., \& Hillier, D. J. 2005, A\&A, 436, 1049

Maschberger, T., \& Kroupa, P. 2009, MNRAS, 395, 931

Massey, P. \& Johnson, J. 1993, AJ, 105, 980

McClure, M. 2009, ApJ, 693, L81

Megeath, S. T., Cox, P., Bronfman, L., \& Roelfsema, P. R. 1996, A\&A, 305, 296

Monnier, J. D., \& Millan-Gabet, R. 2002, ApJ, 579, 694

Muench, A. A., Lada, E. A., 2000, ApJ, 533, 358

Muench, A. A., Lada, E. A., Lada, C. J. \& Alves, J. 2002, ApJ, 573,366

Murray, N. \& Rahman, M. 2010, ApJ, 709, 424

Povich, M. S. et al. 2009, ApJ, 696, 1278

Povich, M. S. \& Whitney, B. A. 2010, ApJ, 714, L285 (PW10)

Povich, M. S. et al. 2011, ApJS, in press (CCCP Massive Stars Candidates Paper; P11)

Preibisch, T. et al. 2005, ApJS, 160, 401

Preibisch, T. et al. 2011, ApJS, in press (CCCP Hawk-I paper)

Price, S. D., Egan, M. P., Carey, S. J., Mizuno, D. R., \& Kuchar, T. A. 2001, AJ, 121, 2842

Prisinzano, L. et al. 2008, ApJ, 677, 401
Rathborne, J. M., Brooks, K. J., Burton, M. G., Cohen, M., \& Bontemps, S. 2004, A\&A, 418, 563

Richer, J. S., Shepherd, D. S., Cabrit, S., Bachiller, R., \& Churchwell, E. 2000, in Protostars and Planets IV, eds. V. Mannings, A. Boss, \& S. Russell (Tucson: Univ. Arizona Press), 867

Rieke, G. H. et al. 2004, ApJS, 154, 25

Robitaille, T. P., Whitney, B. A., Indebetouw, R., Wood, K., \& Denzmore, P. 2006, ApJS, 167, 256 (RW06)

Robitaille, T. P., Whitney, B. A., Indebetouw, R., \& Wood, K. 2007, ApJS, 169, 328

Robitaille, T. P. et al. 2008, AJ, 136, 2413

Robitaille, T. P. \& Whitney, B. A. 2010, ApJ, 710, L11

Rosner, R., Musielak, Z. E., Cattaneo, F., Moore, R. L., \& Suess, S. T. 1995, ApJ, 442, L25

Sabbi, E., et al. 2007, AJ, 133, 44

Sana, H., Momany, Y., Gieles, M., Carraro, G., Beletsky, Y., Ivanov, V. D., de Silva, G., \& James, G. 2010, A\&A, 515, 26

Shepherd, D. S. et al. 2007, ApJ, 669, 464

Siess, L., Dufour, E., \& Forestini, M. 2000, A\&A, 358, 593

Skrutskie, M. F. et al. 2006, AJ, 131, 1163

Smith, N., Egan, M. P., Carey, S., Price, S. D., Morse, J. A., \& Price, Paul A. 2000, ApJ, 532, L145

Smith, N., Bally, J., \& Morse, J. A. 2003, ApJ, 587, L105

Smith, N., Stassun, K. G., \& Bally, J. 2005, AJ, 129, 888

Smith, N. 2006a, MNRAS, 367, 763

Smith, N. 2006b, ApJ, 644, 1151

Smith, N., \& Brooks, K. J. 2007, MNRAS, 379, 1279

Smith, N., \& Brooks, K. J. 2008, Handbook of Star Forming

Regions, Volume II: The Southern Sky, ed. B. Reipurth, p.138

Smith, N., Bally, J., \& Walborn, N. R. 2010a, MNRAS, 405, 1153

Smith, N. et al. 2010b, MNRAS, 406, 952 (SP10)

Stassun, K. G., Ardila, D. R., Barsony, M., Basri, G., \& Mathieu, R. D. 2004, AJ, 127, 3537

Stelzer, B., Huélamo, N., Micela, G., \& Hubrig, S. 2006, A\&A, 452, 1001

Stetson, P. 1987, PASP, 99, 191

Telleschi, A., Güdel, M., Briggs, K. R., Audard, M., \& Palla, F., 2007, A\&A, 468, 425

Townsley, L. K. et al. 2011, ApJS, in press (CCCP Intro Paper)

Wang, J. et al. 2011, ApJS, in press (CCCP Tr15 Paper)

Wolk, S. J. et al. ApJS, in press (CCCP Tr16 Paper)

Weidner, C., \& Vink, J. 2010, A\&A, in press (arXiv:1010.2204)

Weingartner, J. C. \& Draine, B. T. 2001, ApJ, 548, 296

Whitney, B. A., Wood, K., Bjorkman, J. E., \& Cohen, M. 2003, ApJ, 598, 1079

Whitney, B. A. et al. 2008, AJ, 136, 18

Yonekura, Y. et al. 2005, ApJ, 634, 476

Zasowski, G., et al. 2009, ApJ, 707, 510 
Table 2

Pan-Carina YSO Catalog: Basic IR Observational Data ${ }^{a}$

\begin{tabular}{|c|c|c|c|c|c|c|c|c|c|c|c|c|c|}
\hline $\begin{array}{c}(1) \\
\text { PCYC } \\
\text { No. }\end{array}$ & $\begin{array}{c}(2) \\
\text { RA } \\
\text { (J2000) }\end{array}$ & $\begin{array}{c}(3) \\
\text { Dec } \\
(\mathrm{J} 2000)\end{array}$ & $\begin{array}{c}(4) \\
\text { SSTGLM }^{\mathrm{b}}\end{array}$ & $\begin{array}{c}(5) \\
J \\
(\mathrm{mag})\end{array}$ & $\begin{array}{c}\stackrel{(6)}{H} \\
(\mathrm{mag})\end{array}$ & $\begin{array}{l}(7) \\
K_{S} \\
(\mathrm{mag})\end{array}$ & $\begin{array}{c}(8) \\
{[3.6]} \\
(\mathrm{mag})\end{array}$ & $\begin{array}{c}(9) \\
{[4.5]} \\
(\mathrm{mag})\end{array}$ & $\begin{array}{c}(10) \\
{[5.8]} \\
(\mathrm{mag})\end{array}$ & $\begin{array}{c}(11) \\
{[8.0]} \\
(\mathrm{mag})\end{array}$ & $\begin{array}{c}(12) \\
{[24]} \\
(\mathrm{mag})\end{array}$ & $\begin{array}{c}(13)-(20) \\
\text { Quality } \\
\text { Flags }{ }^{c}\end{array}$ & $\begin{array}{c}(21) \\
M S X ?\end{array}$ \\
\hline 117 & 104241.56 & -592443.3 & C G287.2040-00.5300 & & 14.5 & 13.2 & 10.7 & 10.0 & 9.3 & 8.8 & 4.3 & 01111113 & \\
\hline 118 & 104241.91 & -592452.7 & C G287.2059-00.5320 & 014.2 & 13.8 & 13.6 & 13.2 & 12.6 & 11.5 & & 5.3 & 11111101 & \\
\hline 119 & 104243.34 & -592506.5 & C G287.2104-00.5339 & $9 \cdots$ & 14.6 & 13.7 & 12.4 & 11.9 & 11.4 & 10.2 & 6.0 & 01111113 & \\
\hline 122 & 104244.22 & -592520.8 & C G287.2139-00.5365 & 515.9 & 14.5 & 13.8 & 12.8 & 12.4 & 11.6 & 10.5 & 4.6 & 11111113 & \\
\hline 124 & 104245.36 & -592525.9 & C G287.2167-00.5366 & & & 14.3 & 12.7 & 12.2 & 12.0 & 10.6 & 4.6 & 00111113 & \\
\hline 125 & 104245.38 & $\begin{array}{lll}-59 & 2513.7\end{array}$ & C G287.2151-00.5336 & $\begin{array}{ll}6 & 15.4\end{array}$ & 14.0 & 13.2 & 12.1 & 11.6 & 11.4 & 10.2 & 4.4 & 11111113 & \\
\hline 126 & 104245.40 & $\begin{array}{lll}-59 & 2539.2\end{array}$ & C G287.2185-00.5399 & $9 \quad \cdots$ & $\ldots$ & $\cdots$ & 13.2 & 12.5 & 11.8 & 10.8 & 4.8 & 00011113 & \\
\hline 129 & 104246.29 & -592531.4 & C G287.2192-00.5370 & $0 \quad \ldots$ & $\ldots$ & $\ldots$ & 13.7 & 11.7 & 10.8 & 10.3 & 3.0 & 00011111 & \\
\hline 133 & 104246.95 & -592520.9 & C G287.2190-00.5338 & $8 \quad \ldots$ & $\ldots$ & 15.0 & 13.8 & 12.2 & 11.3 & & 2.9 & 00111101 & \\
\hline 138 & 104248.09 & -592539.4 & C G287.2236-00.5372 & $2 \cdots$ & 14.1 & 13.2 & 12.2 & 11.7 & 11.3 & 10.5 & 2.8 & 01111111 & \\
\hline 139 & 104248.14 & -592529.0 & C G287.2223-00.5346 & $\begin{array}{ll}6 & 12.6\end{array}$ & 11.6 & 10.1 & 7.6 & 6.9 & 6.0 & 5.1 & $\cdots$ & 11111110 & \\
\hline 141 & 104248.58 & -592538.3 & C G287.2244-00.5364 & $4 \quad \cdots$ & & 14.4 & 12.1 & 11.3 & 10.6 & 10.0 & 3.0 & 00111111 & \\
\hline 144 & 104249.74 & -592536.5 & C G287.2263-00.5348 & $8 \ldots$ & 14.4 & 13.6 & 11.7 & 11.1 & 10.7 & 9.9 & 4.1 & 01111113 & \\
\hline 145 & 104250.47 & -592543.8 & C G287.2286-00.5359 & 915.6 & 13.5 & 11.8 & 9.8 & 9.1 & 8.5 & 7.7 & 4.1 & 11111113 & \\
\hline 147 & 104250.62 & -592525.4 & C G287.2265-00.5312 & 214.4 & 11.6 & 9.5 & 7.4 & 6.7 & 6.1 & 5.1 & 2.0 & 11111111 & \\
\hline 148 & 104251.30 & -592536.0 & C G287.2291-00.5331 & 115.7 & 14.7 & 14.0 & 13.3 & 12.6 & 11.6 & 10.3 & 4.5 & 11111113 & \\
\hline 155 & 104254.93 & -592546.2 & C G287.2372-00.5320 & 015.6 & 14.2 & 13.3 & 12.4 & 12.0 & 11.7 & 10.6 & 5.1 & 11111113 & \\
\hline 156 & 104255.41 & -592456.5 & C G287.2316-00.5194 & 416.6 & 15.4 & 14.4 & 12.8 & 12.3 & 11.6 & 10.9 & 4.5 & 11111113 & \\
\hline 159 & 104258.43 & -592452.6 & C G287.2367-00.5154 & 414.8 & 13.3 & 12.7 & 12.1 & 12.2 & 11.7 & 11.2 & 4.6 & 11111113 & \\
\hline 161 & 104258.67 & -592534.9 & C G287.2427-00.5255 & $5 \ldots$ & $\cdots$ & $\ldots$ & 13.2 & 12.3 & 11.5 & 10.8 & 4.5 & 00011113 & \\
\hline 162 & 104258.78 & -592504.1 & C G287.2389-00.5178 & $8 \quad \ldots$ & $\ldots$ & $\ldots$ & 12.6 & 12.0 & 11.3 & 10.0 & 4.7 & 00011113 & \\
\hline 164 & 104300.32 & $\begin{array}{llll}-59 & 25 & 27.2\end{array}$ & C G287.2448-00.5220 & $\begin{array}{lll}0 & 16.0\end{array}$ & 14.7 & 13.7 & 12.2 & 11.8 & 11.5 & 10.7 & 4.4 & 11111113 & \\
\hline 177 & 104307.36 & -592433.4 & C G287.2509-00.5017 & 715.8 & 13.9 & 12.5 & 10.8 & 10.3 & 9.9 & 9.4 & 4.8 & 11111113 & \\
\hline 179 & 104309.72 & -592455.3 & C G287.2582-00.5047 & & & & 11.3 & 9.0 & 7.0 & 5.9 & 2.2 & 00011111 & \\
\hline 183 & 104311.37 & -592506.1 & C G287.2627-00.5057 & $\begin{array}{ll}7 & 15.7\end{array}$ & 14.3 & 13.8 & 12.7 & 12.4 & 11.8 & 11.2 & 4.9 & 11111113 & \\
\hline 184 & 104311.72 & -592439.3 & C G287.2598-00.4988 & & & & 14.8 & 12.9 & 11.8 & 10.9 & 5.5 & 00011113 & \\
\hline 185 & 104311.91 & -592452.7 & C G287.2619-00.5019 & 9 & & . & 13.7 & 12.4 & 11.6 & 11.0 & 4.9 & 00011113 & \\
\hline 186 & 104312.06 & -592458.8 & C G287.2630-00.5032 & 215.3 & 13.9 & 13.2 & 12.0 & 11.6 & 10.9 & 10.2 & 4.8 & 11111113 & \\
\hline 187 & 104312.22 & -592418.1 & C G287.2580-00.4931 & & & $\ldots$ & 13.6 & 12.6 & 11.6 & 10.4 & 3.7 & 00011113 & \\
\hline 189 & $1043 \quad 12.62$ & $\begin{array}{lll}-59 & 25 & 15.1\end{array}$ & C G287.2662-00.5067 & $7 \quad 16.1$ & 14.7 & .. & 12.6 & 12.1 & 11.8 & 11.3 & 3.6 & 11011113 & \\
\hline 192 & 104314.00 & -592353.8 & C G287.2581-00.4854 & 414.3 & 13.5 & 13.0 & 12.0 & 11.6 & 11.1 & 10.4 & 4.4 & 11111113 & \\
\hline 194 & 104314.24 & -592509.0 & C G287.2684-00.5035 & 516.5 & 15.2 & 14.3 & 13.2 & 12.6 & 12.1 & 11.2 & 3.7 & 11111113 & \\
\hline 197 & 104314.66 & -592423.4 & C G287.2632-00.4920 & 016.4 & 15.2 & 14.6 & 13.6 & 13.0 & 12.1 & 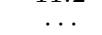 & 4.7 & 11111103 & \\
\hline 372 & 104355.75 & -590848.3 & C G287.2180-00.2216 & & & & 13.0 & 12.1 & 11.4 & 10.6 & 6.5 & 00011113 & \\
\hline 391 & 104358.38 & -590843.3 & C G287.2223-00.2178 & $8 \cdots$ & .. & 14.6 & 13.5 & 12.1 & 11.2 & 10.4 & 5.6 & 00111113 & \\
\hline 400 & 104400.23 & -590854.3 & C G287.2272-00.2186 & $6 \ldots$ & $\ldots$ & $\ldots$ & 12.6 & 10.7 & 9.3 & 8.0 & 3.8 & 00011111 & \\
\hline 407 & 104400.85 & -590856.3 & C G287.2287-00.2185 & $5 \ldots$ & $\ldots$ & $\ldots$ & 13.2 & 12.2 & 10.9 & 9.9 & 5.1 & 00011111 & \\
\hline 416 & 104402.55 & -590824.5 & C G287.2277-00.2090 & $0 \quad \ldots$ & $\ldots$ & $\ldots$ & 12.8 & 12.3 & 11.3 & 10.5 & 6.8 & 00011113 & \\
\hline 417 & 104402.77 & -590849.4 & C G287.2314-00.2148 & $8 \cdots$ & $\ldots$ & $\ldots$ & 13.1 & 11.5 & 10.5 & 9.8 & 5.4 & 00011113 & \\
\hline 426 & 104404.89 & -590756.5 & C G287.2285-00.1998 & 813.6 & 13.0 & 12.8 & 12.7 & 12.6 & 12.0 & 10.9 & 6.2 & 11111113 & \\
\hline 428 & 104404.96 & -590833.3 & C G287.2334-00.2087 & $7 \ldots$ & 15.4 & 14.3 & 13.0 & 12.6 & 12.0 & 11.4 & 7.5 & 01111113 & \\
\hline 438 & 104407.04 & $\begin{array}{llll}-59 & 08 & 39.2\end{array}$ & C G287.2381-00.2081 & $1 \ldots$ & 14.1 & 12.6 & 10.6 & 9.4 & 7.9 & 6.6 & 2.5 & 01111111 & \\
\hline 442 & 104408.12 & -590811.2 & C G287.2365-00.2001 & $1 \ldots$ & 15.4 & 14.4 & 13.3 & 12.8 & 11.8 & $\ldots$ & 5.8 & 01111103 & \\
\hline 528 & 104425.99 & -593338.8 & C G287.4690-00.5571 & 113.6 & 13.0 & 12.6 & 11.9 & 11.6 & 11.4 & 10.8 & $\cdots$ & 11111110 & \\
\hline 556 & 104431.11 & -593310.1 & C G287.4748-00.5450 & & & & 8.8 & 7.4 & 6.0 & 5.0 & $\ldots$ & 00011110 & \\
\hline 563 & 104432.21 & -593359.6 & C G287.4833-00.5561 & 111.0 & 10.8 & 10.8 & 10.7 & 10.7 & 10.4 & 9. & $\ldots$ & 11111110 & \\
\hline 574 & 104433.07 & -593433.2 & C G287.4893-00.5635 & $5 \ldots$ & $\ldots$ & & 13.6 & 13.6 & 11.4 & 10.0 & $\ldots$ & 00011110 & \\
\hline 575 & 104433.18 & -593349.1 & C G287.4837-00.5526 & $\begin{array}{ll}6 & 13.4 \\
\end{array}$ & 12.1 & 11.3 & 10.1 & 9.7 & 9.3 & 8.7 & $\ldots$ & 11111110 & \\
\hline 600 & 104437.01 & -593432.5 & C G287.4965-00.5594 & 412.5 & 12.1 & 11.6 & 10.7 & 10.1 & 9. & 8. & $\ldots$ & 11111110 & \\
\hline 612 & 104438.82 & -593347.2 & C G287.4940-00.5465 & $\begin{array}{ll}5 & 12.0\end{array}$ & 10.9 & & 8.1 & 7.6 & 7.2 & 6.5 & $\ldots$ & 11011110 & \\
\hline 616 & 104439.01 & -593430.6 & C G287.5000-00.5570 & $0 \quad 15.9$ & 14.8 & 14.2 & 13.3 & 12.9 & 12.1 & 11.3 & $\ldots$ & 11111110 & \\
\hline 619 & 104439.79 & -593240.5 & C G287.4871-00.5292 & 214.7 & 13.9 & 13.6 & 12.9 & 12.4 & 11.6 & 10.3 & $\ldots$ & 11111110 & \\
\hline 621 & 104440.03 & -593346.5 & C G287.4962-00.5451 & $1 \ldots$ & $\ldots$ & 13.9 & 12.8 & 12.3 & 11.6 & tente & 3.2 & 00111103 & \\
\hline 625 & 104440.56 & -593354.3 & C G287.4982-00.5465 & $5 \ldots$ & $\ldots$ & $\ldots$ & 12.8 & 12.5 & 12.0 & 11.0 & 2.8 & 00011113 & \\
\hline 634 & 104441.46 & -593356.4 & C G287.5001-00.5462 & $2 \ldots$ & $\ldots$ & $\ldots$ & 12.0 & 11.3 & 11.0 & 10.2 & 2.8 & 00011113 & \\
\hline 642 & 104442.23 & -593335.6 & C G287.4989-00.5403 & 315.2 & 14.2 & 13.3 & 12.0 & 11.4 & 11.0 & & 4.0 & 11111103 & \\
\hline 654 & 104444.05 & -593410.4 & C G287.5068-00.5470 & $0 \ldots$ & $\ldots$ & $\ldots$ & 13.7 & 12.8 & 11.7 & 10.4 & 2.3 & 00011113 & \\
\hline 655 & 104444.06 & -593426.2 & C G287.5088-00.5509 & 915.1 & 13.1 & 11.7 & 10.2 & 9.3 & 8. & 7.4 & & 11111110 & \\
\hline 656 & 104444.09 & -593407.2 & C G287.5064-00.5462 & $2 \cdots$ & $\cdots$ & & 13.4 & 12.1 & 11.1 & 9.7 & 2.5 & 00011113 & \\
\hline 657 & 104444.23 & -593405.1 & C G287.5064-00.5456 & $6 \ldots$ & $\ldots$ & .. & 12.6 & 11.5 & 10.5 & 9.6 & 2.6 & 00011113 & \\
\hline 660 & 104444.41 & -593235.3 & C G287.4951-00.5234 & 415.5 & 14.3 & 13.4 & 12.5 & 12.1 & 11.6 & ... & 2.5 & 11111103 & \\
\hline 699 & 104451.89 & $\begin{array}{llll}-60 & 25 & 11.7\end{array}$ & C G287.9186-01.2913 & $3 \cdots$ & 15.0 & 14.0 & 11.9 & 10.9 & 10.1 & 9.1 & 5.3 & 01111111 & \\
\hline 1295 & 104733.40 & -602656.7 & C G288.2265-01.1635 & 511.5 & 11.2 & 11.0 & 10.8 & 10.8 & 10.5 & 10.1 & 4.5 & 11111113 & \\
\hline 1297 & 104735.69 & -602628.5 & C G288.2271-01.1544 & 414.5 & 13.4 & 12.6 & 11.3 & 10.7 & 9.9 & 8.8 & 4.1 & 11111111 & \\
\hline 1302 & 104742.09 & -602523.1 & C G288.2305-01.1322 & 216.3 & 14.8 & 13.7 & 11.3 & 11.0 & 10.3 & 9.5 & 4.9 & 11111113 & \\
\hline 1304 & 104744.89 & -602726.9 & C G288.2514-01.1602 & $2 \cdots$ & $\ldots$ & & 13.6 & 12.4 & 11.5 & 9.9 & 6.1 & 00011113 & \\
\hline 1308 & 104747.19 & -602633.5 & C G288.2488-01.1448 & $8 \cdots$ & $\ldots$ & 14.5 & 12.2 & 11.5 & 10.9 & 9.9 & 5.3 & 00111113 & \\
\hline 1312 & 104748.52 & -602609.5 & C G288.2482-01.1376 & $6 \ldots$ & 14.4 & 12.7 & 10.4 & 9.7 & 9.1 & 8.3 & 5.1 & 01111111 & \\
\hline 1313 & 104748.97 & -602544.8 & C G288.2458-01.1311 & $1 \ldots$ & $\ldots$ & 14.2 & 12.7 & 12.2 & 11.4 & 10.3 & 7.4 & 00111113 & \\
\hline 1315 & 104750.24 & -602618.6 & C G288.2525-01.1383 & $3 \ldots$ & $\ldots$ & 13.6 & 12.3 & 11.0 & 9.9 & 9.2 & 4.3 & 00111111 & \\
\hline 1316 & 104750.59 & -602704.7 & C G288.2589-01.1494 & 416.1 & 14.0 & $\ldots$ & 12.2 & 12.2 & 11.8 & 10.8 & 6.0 & 11011113 & \\
\hline 1317 & 104750.70 & -602618.1 & C G288.2532-01.1377 & $7 \ldots$ & $\ldots$ & $\ldots$ & 12.8 & 10.8 & 9.5 & 8.6 & 4.5 & 00011111 & \\
\hline 1319 & 104751.67 & $\begin{array}{llll}-60 & 26 & 23.9\end{array}$ & C G288.2557-01.1383 & $3 \cdots$ & $\cdots$ & 14.0 & 12.7 & 11.8 & 11.3 & 10.6 & 4.7 & 00111111 & \\
\hline
\end{tabular}


Table 2 - Continued

\begin{tabular}{|c|c|c|c|c|c|c|c|c|c|c|c|}
\hline $\begin{array}{c}(1) \\
\text { PCYC } \\
\text { No. }\end{array}$ & $\begin{array}{c}(2) \\
\text { RA } \\
(\mathrm{J} 2000)\end{array}$ & $\begin{array}{c}(3) \\
\text { Dec } \\
(\mathrm{J} 2000)\end{array}$ & $\begin{array}{c}\text { (4) } \\
\text { SSTGLM }^{\mathrm{b}}\end{array}$ & $\begin{array}{c}(7) \\
K_{S} \\
(\mathrm{mag})\end{array}$ & $\begin{array}{c}(8) \\
{[3.6]} \\
(\mathrm{mag})\end{array}$ & $\begin{array}{c}(9) \\
{[4.5]} \\
(\mathrm{mag})\end{array}$ & $\begin{array}{c}(10) \\
{[5.8]} \\
(\mathrm{mag})\end{array}$ & $\begin{array}{c}(11) \\
{[8.0]} \\
(\mathrm{mag})\end{array}$ & $\begin{array}{c}(12) \\
{[24]} \\
(\mathrm{mag})\end{array}$ & $\begin{array}{c}\text { (13)-(20) } \\
\text { Quality } \\
\text { Flagsc }^{c}\end{array}$ & \\
\hline 1320 & 104752.07 & -602627.0 & C G288.2569-01.1386 $\ldots$ & $\cdots$ & 12.2 & 10.2 & 9.0 & 8.2 & 4.8 & 00011111 & \\
\hline \multicolumn{12}{|c|}{$\begin{array}{l}\text { Note. - This Table is available in its entirety in a machine-readable form in the online journal. A portion is reproduced here for guidance } \\
\text { regarding its form and content and to highlight sources of interest. Sources are grouped according to their apparent association with the YSO } \\
\text { sub-clusters discussed in } \S 6 \text {. } \\
\text { a The typical photometric uncertainty is } 0.1 \mathrm{mag} \text {. } \\
\text { b Source names are preceded by either a "C" for the highly-reliable Vela-Carina Catalog or an "A" for the more-complete Archive. } \\
\text { c Quality flags are defined as follows: } 0=\text { non-detection, } 1=\text { detection, } 2=\text { flux density lower limit (magnitude upper limit), and } 3=\text { flux density } \\
\text { upper limit (magnitude lower limit). }\end{array}$} \\
\hline
\end{tabular}

Table 3

Pan-Carina YSO Catalog: Modeled Properties and CCCP Matches

\begin{tabular}{|c|c|c|c|c|c|c|c|c|c|c|c|}
\hline $\begin{array}{c}(1) \\
\text { PCYC }\end{array}$ & (2) & (3) & (4) & (5) & (6) & (7) & (8) & $(9)$ & (10) & (11) & $(12)$ \\
\hline $\begin{array}{l}\text { PCYC } \\
\text { No. }\end{array}$ & $\begin{array}{c}\left\langle\log L_{\mathrm{bol}}\right\rangle \\
\left(\mathrm{L}_{\odot}\right)\end{array}$ & $\begin{array}{c}\sigma\left(\log L_{\mathrm{bol}}\right) \\
\left(\mathrm{L}_{\odot}\right)\end{array}$ & $\begin{array}{l}\left\langle M_{\star}\right\rangle \\
\left(\mathrm{M}_{\odot}\right)\end{array}$ & $\begin{array}{c}\sigma\left(M_{\star}\right) \\
\left(\mathrm{M}_{\odot}\right)\end{array}$ & $\begin{array}{l}\left\langle\log \dot{M}_{\text {env }}\right\rangle \\
\left(\mathrm{M}_{\odot} \mathrm{yr}^{-1}\right)\end{array}$ & $\begin{array}{c}\sigma\left(\log \dot{M}_{\text {env }}\right) \\
\left(\mathrm{M}_{\odot} \mathrm{yr}^{-1}\right)\end{array}$ & Stage & $\begin{array}{c}\text { CCCP Match } \\
\text { CXOGNC J }\end{array}$ & $\begin{array}{l}\text { Membe } \\
\mathrm{Cl} / \mathrm{Gr}\end{array}$ & $\begin{array}{l}\operatorname{ship}^{a} \\
\text { Reg }\end{array}$ & Notes $^{b}$ \\
\hline 117 & 1.9 & 0.2 & 3.4 & 1.1 & -4.8 & 1.9 & $0 / \mathrm{I}$ & & & $\mathrm{D}$ & $\mathrm{P}$ \\
\hline 118 & 1.6 & 0.1 & 2.5 & 0.2 & $\ldots$ & $\ldots$ & II & & & $\mathrm{D}$ & $\mathrm{P}$ \\
\hline 119 & 1.3 & 0.9 & 1.9 & 0.9 & -7.1 & 2.5 & II & & & $\mathrm{D}$ & $\mathrm{P}$ \\
\hline 122 & 1.2 & 0.9 & 1.3 & 1.1 & -7.3 & 2.2 & $\mathrm{~A}$ & & & $\mathrm{D}$ & $\mathrm{P}$ \\
\hline 124 & 1.8 & 0.7 & 2.7 & 1.0 & -6.3 & 2.3 & $\mathrm{~A}$ & & $\mathrm{G} 4$ & $\mathrm{D}$ & $\mathrm{P}$ \\
\hline 125 & 1.9 & 0.5 & 2.9 & 0.8 & $\cdots$ & $\cdots$ & III & $104245.34-592513.3$ & & $\mathrm{D}$ & $\mathrm{P}, 3 \mathrm{X}$ \\
\hline 126 & 1.7 & 1.1 & 2.4 & 1.3 & -6.0 & 2.2 & $\mathrm{~A}$ & & G4 & $\mathrm{D}$ & $\mathrm{P}$ \\
\hline 129 & 2.6 & 0.2 & 6.4 & 1.0 & -4.0 & 0.8 & $0 / \mathrm{I}$ & & G4 & $\mathrm{D}$ & $\mathrm{P}$ \\
\hline 133 & 2.3 & 0.4 & 4.0 & 1.3 & -4.2 & 1.8 & $0 / I$ & & G4 & $\mathrm{D}$ & $\mathrm{P}$ \\
\hline 138 & 2.0 & 0.1 & 3.1 & 0.7 & -4.5 & 0.3 & $0 / \mathrm{I}$ & $104248.10-592539.3$ & G4 & $\mathrm{D}$ & $\mathrm{P}$ \\
\hline 139 & 2.6 & 0.3 & 4.9 & 1.2 & -8.3 & 1.8 & II & $104248.17-592528.7$ & G4 & $\mathrm{D}$ & $\mathrm{P}$ \\
\hline 141 & 2.0 & 0.1 & 4.5 & 0.6 & -4.4 & 2.0 & $0 / \mathrm{I}$ & & G4 & $\mathrm{D}$ & $\mathrm{P}$ \\
\hline 144 & 1.3 & 0.3 & 1.6 & 0.7 & -5.4 & 2.3 & $0 / \mathrm{I}$ & & & $\mathrm{D}$ & $\mathrm{P}$ \\
\hline 145 & 2.9 & 1.7 & 4.4 & 1.9 & $\ldots$ & $\ldots$ & II & $104250.50-592544.2$ & & $\mathrm{D}$ & $\mathrm{P}$ \\
\hline 147 & 3.1 & 0.1 & 6.5 & 0.4 & $\ldots$ & $\ldots$ & II & & & $\mathrm{D}$ & $\mathrm{P}$ \\
\hline 148 & 0.7 & 0.5 & 0.6 & 0.5 & -4.8 & 2.2 & $0 / I$ & $104251.41-592535.6$ & & $\mathrm{D}$ & $\mathrm{P}$ \\
\hline 155 & 1.7 & 1.0 & 2.3 & 1.0 & -7.2 & 2.6 & $\mathrm{~A}$ & $104254.93-592545.4$ & & $\mathrm{D}$ & $\mathrm{P}$ \\
\hline 156 & 0.9 & 0.6 & 1.0 & 0.7 & -5.4 & 2.4 & $0 / \mathrm{I}$ & $104255.40-592456.5$ & & $\mathrm{D}$ & $\mathrm{P}$ \\
\hline 159 & 0.9 & 0.4 & 1.6 & 0.9 & -6.5 & 2.6 & $\mathrm{~A}$ & & & $\mathrm{D}$ & $\mathrm{P}$ \\
\hline 161 & 1.7 & 1.0 & 2.4 & 1.3 & -5.3 & 2.1 & $\mathrm{~A}$ & $104258.65-592534.4$ & & $\mathrm{D}$ & $\mathrm{P}$ \\
\hline 162 & 2.0 & 1.2 & 2.6 & 1.5 & $\cdots$ & $\cdots$ & $\mathrm{A}$ & $104258.78-592503.9$ & & $\mathrm{D}$ & $\mathrm{P}$ \\
\hline 164 & 1.3 & 0.4 & 1.8 & 0.8 & $\ldots$ & $\ldots$ & $\mathrm{A}$ & $104300.29-592526.9$ & & $\mathrm{D}$ & $\mathrm{P}$ \\
\hline 177 & 1.7 & 0.2 & 2.6 & 0.4 & $\cdots$ & $\cdots$ & $\mathrm{A}$ & $104307.37-592433.6$ & & $\mathrm{D}$ & $\mathrm{Q}$ \\
\hline 179 & 3.6 & 0.0 & 10.1 & 0.9 & -2.6 & 0.2 & $0 / I$ & $104309.83-592454.2$ & & $\mathrm{D}$ & $\mathrm{Q}, \mathrm{MSX}$ \\
\hline 183 & 0.7 & 0.5 & 1.2 & 0.8 & -7.9 & 2.4 & $\mathrm{~A}$ & & & $\mathrm{D}$ & $\mathrm{Q}$ \\
\hline 184 & 1.8 & 1.1 & 1.7 & 1.6 & -5.4 & 2.0 & $0 / \mathrm{I}$ & & & $\mathrm{D}$ & $\mathrm{Q}$ \\
\hline 185 & 1.8 & 0.6 & 2.7 & 1.5 & -5.2 & 2.0 & $0 / \mathrm{I}$ & & & $\mathrm{D}$ & $\mathrm{Q}$ \\
\hline 186 & 1.1 & 0.2 & 2.0 & 0.8 & $\ldots$ & $\ldots$ & II & $104312.06-592458.5$ & & $\mathrm{D}$ & $\mathrm{Q}$ \\
\hline 187 & 1.6 & 1.1 & 1.9 & 1.3 & -5.4 & 2.0 & $0 / \mathrm{I}$ & & & $\mathrm{D}$ & $\mathrm{Q}$ \\
\hline 189 & 0.9 & 0.5 & 1.2 & 0.7 & -6.3 & 2.5 & $0 / \mathrm{I}$ & $104312.59-592516.2$ & & $\mathrm{D}$ & $\mathrm{Q}$ \\
\hline 192 & 1.2 & 0.3 & 2.1 & 0.5 & $\ldots$ & $\ldots$ & II & $104313.98-592353.0$ & & $\mathrm{D}$ & $\mathrm{Q}$ \\
\hline 194 & 0.8 & 0.6 & 0.8 & 0.9 & -6.1 & 2.4 & $\mathrm{~A}$ & & & $\mathrm{D}$ & $\mathrm{Q}$ \\
\hline 197 & 1.0 & 0.7 & 1.0 & 0.9 & -5.9 & 2.3 & $\mathrm{~A}$ & & & $\mathrm{D}$ & $\mathrm{Q}$ \\
\hline 372 & 2.0 & 1.2 & 3.2 & 1.0 & $\cdots$ & $\cdots$ & II & & & $\mathrm{D}$ & $\mathrm{R}$ \\
\hline 391 & 1.0 & 0.1 & 1.1 & 0.4 & -5.1 & 2.4 & $0 / \mathrm{I}$ & & & $\mathrm{D}$ & $\mathrm{R}$ \\
\hline 400 & 2.4 & 1.4 & 3.2 & 1.8 & -4.4 & 1.6 & $0 / \mathrm{I}$ & & & $\mathrm{D}$ & $\mathrm{R}$ \\
\hline 407 & 1.5 & 1.0 & 1.4 & 1.5 & -5.1 & 2.0 & $0 / \mathrm{I}$ & & & $\mathrm{D}$ & $\mathrm{R}$ \\
\hline 416 & 1.3 & 0.8 & 2.1 & 1.0 & $\cdots$ & $\ldots$ & II & & & $\mathrm{D}$ & $\mathrm{R}$ \\
\hline 417 & 2.2 & 1.3 & 3.3 & 1.4 & -5.9 & 1.8 & $0 / I$ & & & $\mathrm{D}$ & $\mathrm{R}$ \\
\hline 426 & 1.0 & 0.8 & 1.8 & 0.6 & -8.8 & 2.8 & II & & & $\mathrm{D}$ & $\mathrm{R}$ \\
\hline 428 & 0.9 & 0.4 & 1.5 & 0.7 & -7.0 & 2.7 & II & $104404.95-590833.6$ & & $\mathrm{D}$ & $\mathrm{R}$ \\
\hline 438 & 2.1 & 0.3 & 2.8 & 1.1 & -3.8 & 1.5 & $0 / \mathrm{I}$ & & & $\mathrm{D}$ & $\mathrm{R}$ \\
\hline 442 & 1.5 & 1.0 & 1.6 & 1.1 & -6.4 & 2.5 & $\mathrm{~A}$ & & & $\mathrm{D}$ & $\mathrm{R}$ \\
\hline 528 & 1.1 & 0.7 & 2.0 & 0.6 & -7.2 & 2.8 & II & $104425.97-593339.0$ & C5 & $\mathrm{A}$ & Cr 232 \\
\hline 556 & 3.6 & 2.0 & 9.0 & 3.2 & -4.0 & 1.5 & $0 / I$ & $104431.23-593311.2$ & C5 & $\mathrm{A}$ & Cr 232 \\
\hline 563 & 1.7 & 0.0 & 3.7 & 0.2 & -8.0 & 3.0 & II & & C5 & $\mathrm{A}$ & Cr 232 \\
\hline 574 & 2.1 & 1.2 & 3.2 & 2.1 & -4.4 & 1.6 & $0 / I$ & & C5 & $\mathrm{A}$ & Cr 232 \\
\hline 575 & 1.8 & 0.2 & 3.1 & 0.7 & -7.4 & 2.2 & II & $104433.23-593348.4$ & C5 & $\mathrm{A}$ & Cr 232 \\
\hline 600 & 1.6 & 0.2 & 2.6 & 0.3 & $\cdots$ & $\cdots$ & II & & C5 & $\mathrm{A}$ & Cr 232 \\
\hline 612 & 3.4 & 1.9 & 6.4 & 3.0 & $\ldots$ & $\ldots$ & II & & C5 & $\mathrm{A}$ & Cr 232 \\
\hline 616 & 0.9 & 0.7 & 1.1 & 0.9 & -6.6 & 2.1 & $\mathrm{~A}$ & & & $\mathrm{~A}$ & $\mathrm{Cr} 232$ \\
\hline 619 & 1.5 & 1.0 & 1.6 & 1.7 & -7.2 & 2.2 & $\mathrm{~A}$ & $104439.84-593240.2$ & C5 & $\mathrm{A}$ & Cr 232 \\
\hline 621 & 1.4 & 1.0 & 1.6 & 1.2 & -5.9 & 2.2 & $\mathrm{~A}$ & & C5 & $\mathrm{A}$ & Cr 232 \\
\hline 625 & 1.4 & 0.9 & 1.7 & 1.1 & -6.3 & 2.3 & $\mathrm{~A}$ & & C5 & $\mathrm{A}$ & Cr 232 \\
\hline 634 & 1.8 & 1.5 & 2.5 & 1.2 & -5.6 & 2.2 & A & & C5 & A & Cr 232 \\
\hline
\end{tabular}


Table 3 - Continued

\begin{tabular}{|c|c|c|c|c|c|c|c|c|c|c|c|}
\hline $\begin{array}{l}(1) \\
\text { PCYC } \\
\text { No. }\end{array}$ & $\begin{array}{c}(2) \\
\left\langle\log L_{\mathrm{bol}}\right\rangle \\
\left(\mathrm{L}_{\odot}\right)\end{array}$ & $\begin{array}{c}(3) \\
\sigma\left(\log L_{\mathrm{bol}}\right) \\
\left(\mathrm{L}_{\odot}\right)\end{array}$ & $\begin{array}{c}(4) \\
\left\langle M_{\star}\right\rangle \\
\left(\mathrm{M}_{\odot}\right)\end{array}$ & $\begin{array}{c}(5) \\
\sigma\left(M_{\star}\right) \\
\left(\mathrm{M}_{\odot}\right)\end{array}$ & $\begin{array}{c}(6) \\
\left\langle\log \dot{M}_{\text {env }}\right\rangle \\
\left(\mathrm{M}_{\odot} \mathrm{yr}^{-1}\right)\end{array}$ & $\begin{array}{c}(7) \\
\sigma\left(\log \dot{M}_{\text {env }}\right) \\
\left(\mathrm{M}_{\odot} \mathrm{yr}^{-1}\right)\end{array}$ & $\begin{array}{c}(8) \\
\text { Stage }\end{array}$ & $\begin{array}{c}(9) \\
\text { CCCP Match } \\
\text { CXOGNC J }\end{array}$ & $\begin{array}{l}(10) \\
\text { Membe } \\
\text { Cl/Gr }\end{array}$ & $\begin{array}{r}(11) \\
\text { ship }^{\mathrm{a}} \\
\text { Reg }\end{array}$ & $\begin{array}{c}(12) \\
\text { Notes }^{b}\end{array}$ \\
\hline 642 & 1.9 & 0.8 & 2.8 & 0.9 & $\ldots$ & $\ldots$ & A & & C5 & A & Cr 232 \\
\hline 655 & 2.1 & 0.3 & 3.9 & 1.3 & -5.0 & 2.1 & $\mathrm{~A}$ & & & A & Cr 232 \\
\hline 656 & 2.3 & 1.4 & 3.1 & 1.8 & -5.2 & 1.9 & A & & & $\mathrm{A}$ & Cr 232 \\
\hline 657 & 2.2 & 1.3 & 3.3 & 1.6 & -5.3 & 1.9 & A & & & A & Cr 232 \\
\hline 660 & 1.8 & 1.1 & 2.2 & 1.2 & -8.5 & 2.4 & A & & $\mathrm{C} 5$ & A & Cr 232 \\
\hline 1297 & 1.2 & 0.4 & 1.3 & 1.3 & -5.0 & 2.3 & $0 / \mathrm{I}$ & & & $\mathrm{D}$ & $\mathrm{O}$ \\
\hline 1302 & 1.4 & 1.1 & 1.9 & 0.6 & $\ldots$ & $\ldots$ & $\mathrm{A}$ & & & $\mathrm{D}$ & $\mathrm{O}$ \\
\hline 1304 & 2.6 & 1.5 & 4.2 & 1.5 & $\ldots$ & $\ldots$ & $\mathrm{A}$ & & & $\mathrm{D}$ & $\mathrm{O}$ \\
\hline 1308 & 2.0 & 1.2 & 3.1 & 0.9 & $\ldots$ & $\ldots$ & II & & & $\mathrm{D}$ & $\mathrm{O}$ \\
\hline 1312 & 2.2 & 0.1 & 3.7 & 0.4 & $\ldots$ & $\ldots$ & II & $104748.69-602611.1$ & & $\mathrm{D}$ & $\mathrm{O}$ \\
\hline 1313 & 1.3 & 0.8 & 2.8 & 0.6 & $\ldots$ & $\ldots$ & II & & & $\mathrm{D}$ & $\mathrm{O}$ \\
\hline 1315 & 1.5 & 0.4 & 2.1 & 1.2 & -5.0 & 1.9 & $0 / \mathrm{I}$ & $104750.03-602619.1$ & & $\mathrm{D}$ & $\mathrm{O}$ \\
\hline
\end{tabular}

Note. - This Table is available in its entirety in a machine-readable form in the online journal. A portion is shown here for guidance regarding its form and content and to highlight the sources of interest presented in Table 2 and $\S 6$.

a The Membership columns follow F11.

b The Notes column includes the following: O, P, Q, R, or Cr 232 refer to membership in the groups discussed in $\S 6$; $2 \mathrm{X}$ or $3 \mathrm{X}$ indicates that the Spitzer source was matched to 2 or 3 CCCP X-ray sources; and MSX means that the YSO is also an MSX source.

Table 4

Candidate X-ray-Emitting Stage 0/I YSOs

\begin{tabular}{|c|c|c|c|c|c|c|c|c|c|c|c|}
\hline \multirow[b]{3}{*}{$\begin{array}{l}\text { PCYC } \\
\text { No. }\end{array}$} & $(2)$ & (3) & (4) & $(5)$ & (6) & (7) & (8) & \multirow[b]{3}{*}{$\begin{array}{l}\text { CCCP Match } \\
\text { CXOGNC J. }\end{array}$} & $(10)$ & $(11)$ & $(12)$ \\
\hline & \multicolumn{7}{|c|}{ Physical Parameters from YSO Models } & & \multicolumn{3}{|c|}{ Observed X-ray Properties ${ }^{\mathrm{b}}$} \\
\hline & $\begin{array}{l}\left\langle M_{\star}\right\rangle \\
\left(\mathrm{M}_{\odot}\right)\end{array}$ & $\begin{array}{c}\sigma\left(M_{\star}\right) \\
\left(\mathrm{M}_{\odot}\right)\end{array}$ & $\begin{array}{c}\left\langle\log T_{\star}\right\rangle \\
(\mathrm{K})\end{array}$ & $\begin{array}{c}\sigma\left(\log T_{\star}\right) \\
(\mathrm{K})\end{array}$ & $\begin{array}{c}\left\langle\log \frac{\dot{M}_{\mathrm{env}}}{M_{\star}}\right\rangle \\
\left(\mathrm{yr}^{-1^{\star}}\right)\end{array}$ & $\begin{array}{l}\left\langle A_{V, t}\right\rangle \\
(\mathrm{mag})\end{array}$ & $\begin{array}{c}\operatorname{Max}, \sigma\left(A_{V, t}\right)^{\mathrm{a}} \\
(\mathrm{mag})\end{array}$ & & $\begin{array}{c}\text { Net } \\
\text { Counts }\end{array}$ & $\begin{array}{c}E_{\text {median }} \\
(\mathrm{keV})\end{array}$ & $\begin{array}{c}\log F_{t} \\
\left(\mathrm{erg} / \mathrm{cm}^{2} / \mathrm{s}\right)\end{array}$ \\
\hline \multicolumn{12}{|c|}{ Hard X-ray Counterparts $\left(E_{\text {median }}>2 \mathrm{keV}\right)$} \\
\hline 138 & 3.1 & 0.7 & 3.6 & 0.0 & -5.0 & $5.0 \times 10^{2}$ & $4.5 \times 10^{2}$ & $104248.10-592539.3$ & .3101 .5 & 3.3 & -13.4 \\
\hline 140 & 0.8 & 0.9 & 3.5 & 0.1 & -5.8 & $4.1 \times 10^{0}$ & $6.4 \times 10^{2}$ & $104248.50-594835.5$ & $.5 \quad 5.2$ & 2.3 & -14.9 \\
\hline 179 & 10.1 & 0.9 & 3.6 & 0.0 & -3.6 & $7.2 \times 10^{2}$ & $4.9 \times 10^{2}$ & $104309.83-592454.2$ & .244 .9 & 4.0 & -13.9 \\
\hline 239 & 1.2 & 1.2 & 3.6 & 0.2 & -5.6 & $4.6 \times 10^{1}$ & $4.7 \times 10^{2}$ & $104326.70-602622$. & .7149 .6 & 4.7 & -12.9 \\
\hline 282 & 1.9 & 1.5 & 3.7 & 0.2 & -5.6 & $3.1 \times 10^{1}$ & $9.9 \times 10^{4}$ & $104339.83-593300$. & .93 .6 & 3.0 & -14.9 \\
\hline 318 & 2.8 & 1.0 & 3.7 & 0.2 & -5.8 & $1.5 \times 10^{1}$ & $1.5 \times 10^{1}$ & $104347.79-593304$. & .45 .6 & 2.9 & -14.8 \\
\hline 348 & 3.7 & 2.4 & 3.8 & 0.4 & -5.4 & $4.9 \times 10^{1}$ & $4.0 \times 10^{5}$ & $104351.67-593257$ & .09 .6 & 2.1 & -14.4 \\
\hline 353 & 1.9 & 1.1 & 3.7 & 0.1 & -5.8 & $7.0 \times 10^{0}$ & $5.1 \times 10^{3}$ & $104352.89-593921$. & .892 .9 & 2.4 & -13.6 \\
\hline 371 & 4.0 & 1.1 & 3.7 & 0.1 & -5.4 & $2.5 \times 10^{1}$ & $1.7 \times 10^{1}$ & $104355.73-592417$. & $.5 \quad 55.3$ & 2.8 & -13.6 \\
\hline 373 & 3.7 & 1.2 & 3.7 & 0.0 & -5.3 & $2.9 \times 10^{1}$ & $1.3 \times 10^{1}$ & $104355.80-592421$. & .711 .8 & 3.2 & -14.1 \\
\hline 387 & 1.5 & 1.7 & 3.6 & 0.2 & -5.4 & $5.7 \times 10^{1}$ & $1.7 \times 10^{3}$ & $104357.77-592833.3$ & .38 .5 & 2.2 & -14.6 \\
\hline 419 & 2.8 & 1.6 & 3.6 & 0.1 & -4.8 & $1.8 \times 10^{2}$ & $1.6 \times 10^{5}$ & $104403.08-593748.7$ & .719 .0 & 2.6 & -14.5 \\
\hline 484 & 9.5 & 0.8 & 4.0 & 0.6 & -5.2 & $4.1 \times 10^{1}$ & $3.5 \times 10^{1}$ & $104417.86-602745$. & .95 .5 & 4.7 & -14.4 \\
\hline 598 & 2.2 & 1.6 & 3.7 & 0.3 & -5.3 & $3.7 \times 10^{1}$ & $1.0 \times 10^{3}$ & $104437.14-595802$ & $.4 \quad 29.1$ & 4.0 & -13.9 \\
\hline 623 & 3.5 & 1.3 & 3.8 & 0.5 & -4.9 & $4.1 \times 10^{1}$ & $9.3 \times 10^{1}$ & $104440.31-594339.7$ & $\begin{array}{ll}.7 & 2.3\end{array}$ & 5.4 & -14.9 \\
\hline 686 & 0.9 & 1.0 & 3.6 & 0.1 & $\ldots$ & $5.2 \times 10^{0}$ & $1.5 \times 10^{2}$ & $104449.16-595346$ & 6138.3 & 2.9 & -13.5 \\
\hline 699 & 0.7 & 1.0 & 3.5 & 0.1 & -4.7 & $3.0 \times 10^{1}$ & $1.4 \times 10^{3}$ & $104451.91-602511$. & .9376 .8 & 5.2 & -12.3 \\
\hline 713 & 1.6 & 0.7 & 3.7 & 0.1 & -6.2 & $8.9 \times 10^{0}$ & $7.0 \times 10^{0}$ & $104455.40-592634$. & .535 .5 & 4.4 & -14.1 \\
\hline 774 & 2.1 & 1.1 & 3.7 & 0.1 & -6.6 & $1.5 \times 10^{1}$ & $1.6 \times 10^{1}$ & $104507.15-600156$. & .979 .2 & 3.0 & -13.8 \\
\hline 897 & 0.6 & 0.5 & 3.6 & 0.1 & -5.3 & $3.2 \times 10^{1}$ & $7.3 \times 10^{2}$ & $104530.83-595809$. & $.5 \quad 5.3$ & 2.2 & -15.0 \\
\hline 1005 & 2.4 & 0.9 & 3.7 & 0.1 & -9.0 & $1.5 \times 10^{1}$ & $8.9 \times 10^{0}$ & $104549.19-601637.3$ & .319 .4 & 3.0 & -14.3 \\
\hline 1073 & 1.7 & 1.0 & 3.6 & 0.0 & -5.7 & $8.4 \times 10^{0}$ & $4.9 \times 10^{0}$ & $104603.53-595339.3$ & .328 .6 & 2.2 & -14.3 \\
\hline 1185 & 1.0 & 0.9 & 3.7 & 0.2 & -5.8 & $1.5 \times 10^{1}$ & $2.6 \times 10^{1}$ & $104637.74-594804.4$ & .415 .9 & 2.2 & -14.6 \\
\hline 1236 & 2.7 & 0.4 & 3.6 & 0.0 & -5.0 & $2.8 \times 10^{1}$ & $6.1 \times 10^{0}$ & $104659.85-602535$ & $.0 \quad 10.3$ & 2.3 & -14.4 \\
\hline 1315 & 2.1 & 1.2 & 3.6 & 0.0 & -5.3 & $2.7 \times 10^{1}$ & $1.6 \times 10^{1}$ & $104750.03-602619$ & .117 .2 & 2.7 & -14.0 \\
\hline 1367 & 7.6 & 2.5 & 4.0 & 0.2 & -5.4 & $5.6 \times 10^{2}$ & $1.7 \times 10^{6}$ & $104824.37-600759.5$ & .522 .0 & 4.2 & -13.9 \\
\hline \multicolumn{12}{|c|}{ Soft X-ray Counterparts $\left(E_{\text {median }} \leq 2 \mathrm{keV}\right)$} \\
\hline 83 & 0.8 & 0.6 & 3.6 & 0.0 & -5.1 & $2.1 \times 10^{1}$ & $2.9 \times 10^{1}$ & $104216.00-593607.6$ & .613 .5 & 1.5 & -14.4 \\
\hline 148 & 0.6 & 0.5 & 3.6 & 0.1 & -4.5 & $4.6 \times 10^{1}$ & $1.4 \times 10^{2}$ & $104251.41-592535.6$ & $6 \quad 5.5$ & 1.5 & -14.9 \\
\hline
\end{tabular}


A Pan-Carina YSO Catalog

Table 4 - Continued

\begin{tabular}{|c|c|c|c|c|c|c|c|c|c|c|c|}
\hline \multirow[t]{2}{*}{$(1)$} & (2) & (3) & $(4)$ & $(5)$ & (6) & (7) & (8) & \multirow[b]{3}{*}{$\begin{array}{l}\text { CCCP Match } \\
\text { CXOGNC J. }\end{array}$} & $(10)$ & $(11)$ & $(12)$ \\
\hline & \multicolumn{7}{|c|}{ Physical Parameters from YSO Models } & & \multicolumn{3}{|c|}{ Observed X-ray Properties ${ }^{\mathrm{b}}$} \\
\hline $\begin{array}{l}\text { PCYC } \\
\text { No. }\end{array}$ & $\begin{array}{l}\left\langle M_{\star}\right\rangle \\
\left(\mathrm{M}_{\odot}\right)\end{array}$ & $\begin{array}{c}\sigma\left(M_{\star}\right) \\
\left(\mathrm{M}_{\odot}\right)\end{array}$ & $\begin{array}{c}\left\langle\log T_{\star}\right\rangle \\
(\mathrm{K})\end{array}$ & $\begin{array}{c}\sigma\left(\log T_{\star}\right) \\
(\mathrm{K})\end{array}$ & $\begin{array}{c}\left\langle\log \frac{\dot{M}_{\text {env }}}{M_{\star}}\right\rangle \\
\left(\mathrm{yr}^{-1}\right)\end{array}$ & $\begin{array}{l}\left\langle A_{V, t}\right\rangle \\
\text { (mag) }\end{array}$ & $\begin{array}{c}\operatorname{Max}, \sigma\left(A_{V, t}\right)^{\mathrm{a}} \\
(\mathrm{mag})\end{array}$ & & $\begin{array}{l}\text { Net } \\
\text { Counts }\end{array}$ & $\begin{array}{c}E_{\text {median }} \\
(\mathrm{keV})\end{array}$ & $\begin{array}{c}\log F_{t} \\
\left(\mathrm{erg} / \mathrm{cm}^{2} / \mathrm{s}\right)\end{array}$ \\
\hline 156 & 1.0 & 0.7 & 3.7 & 0.2 & -5.4 & $2.8 \times 10^{1}$ & $2.9 \times 10^{2}$ & $104255.40-592456.5$ & $5 \quad 3.6$ & 1.5 & -14.9 \\
\hline 189 & 1.2 & 0.7 & 3.6 & 0.1 & -6.3 & $1.0 \times 10^{1}$ & $3.3 \times 10^{1}$ & $104312.59-592516.2$ & 230.1 & 1.8 & -14.4 \\
\hline 288 & 2.6 & 1.0 & 3.6 & 0.0 & -5.1 & $2.5 \times 10^{2}$ & $5.4 \times 10^{3}$ & $104341.07-594537.2$ & 251.1 & 1.5 & -14.4 \\
\hline 346 & 2.5 & 0.6 & 3.6 & 0.0 & -4.7 & $3.1 \times 10^{2}$ & $6.6 \times 10^{2}$ & $104351.59-600525.2$ & 217.0 & 1.3 & -14.6 \\
\hline 352 & 1.7 & 1.6 & 3.6 & 0.2 & -5.7 & $2.3 \times 10^{1}$ & $6.5 \times 10^{4}$ & $104352.58-593025.1$ & 18.7 & 1.4 & -15.1 \\
\hline 355 & 2.0 & 0.9 & 3.7 & 0.1 & -5.3 & $3.2 \times 10^{1}$ & $5.0 \times 10^{4}$ & $104353.56-593410.1$ & 15.6 & 1.5 & -15.0 \\
\hline 369 & 2.1 & 1.5 & 3.7 & 0.1 & -6.3 & $4.4 \times 10^{0}$ & $1.0 \times 10^{3}$ & $104355.20-600519.8$ & 811.5 & 1.2 & -14.4 \\
\hline 376 & 0.5 & 0.7 & 3.6 & 0.3 & $\ldots$ & $4.8 \times 10^{0}$ & $4.2 \times 10^{1}$ & $104355.92-592537.6$ & $6 \quad 19.5$ & 1.5 & -14.7 \\
\hline 424 & 2.8 & 1.1 & 3.7 & 0.2 & -5.5 & $5.6 \times 10^{0}$ & $5.5 \times 10^{0}$ & $104403.87-593308.3$ & 34.7 & 1.0 & -15.4 \\
\hline 432 & 2.3 & 0.9 & 3.7 & 0.0 & -5.3 & $1.2 \times 10^{2}$ & $2.0 \times 10^{2}$ & $104406.04-595510.6$ & $6 \quad 11.1$ & 1.7 & -14.6 \\
\hline 450 & 2.3 & 1.2 & 3.7 & 0.2 & -5.7 & $2.8 \times 10^{0}$ & $1.8 \times 10^{1}$ & $104409.95-593436.1$ & 127.2 & 1.5 & -14.3 \\
\hline 459 & 1.3 & 0.7 & 3.6 & 0.1 & -7.2 & $8.7 \times 10^{0}$ & $5.4 \times 10^{1}$ & $104411.75-592431.1$ & 119.5 & 1.6 & -14.5 \\
\hline 496 & 1.7 & 1.4 & 3.6 & 0.1 & -5.3 & $4.5 \times 10^{1}$ & $1.6 \times 10^{2}$ & $104421.14-593000.9$ & 94.6 & 1.3 & -15.1 \\
\hline 505 & 0.8 & 1.0 & 3.6 & 0.1 & -6.2 & $3.4 \times 10^{0}$ & $3.1 \times 10^{2}$ & $104423.08-600819.3$ & 326.9 & 1.2 & -14.4 \\
\hline 508 & 0.7 & 0.9 & 3.6 & 0.2 & -5.8 & $5.8 \times 10^{0}$ & $6.3 \times 10^{2}$ & $104423.32-592513.5$ & $5 \quad 5.5$ & 1.6 & -15.1 \\
\hline 521 & 1.3 & 1.1 & 3.7 & 0.3 & -8.3 & $5.1 \times 10^{0}$ & $2.0 \times 10^{3}$ & $104425.68-593923.5$ & $5 \quad 55.1$ & 1.3 & -14.5 \\
\hline 548 & 1.7 & 1.7 & 3.6 & 0.1 & -5.4 & $5.7 \times 10^{1}$ & $6.7 \times 10^{2}$ & $104429.88-594533.8$ & $8 \quad 4.6$ & 1.3 & -15.2 \\
\hline 556 & 9.0 & 3.2 & 4.0 & 0.6 & -4.9 & $8.7 \times 10^{1}$ & $2.4 \times 10^{3}$ & $104431.23-593311.2$ & $2 \quad 18.2$ & 1.9 & -14.3 \\
\hline 593 & 1.5 & 1.2 & 3.7 & 0.2 & -7.7 & $5.6 \times 10^{0}$ & $1.1 \times 10^{4}$ & $104436.06-594258.4$ & $4 \quad 12.9$ & 1.5 & -14.7 \\
\hline 667 & 1.5 & 0.8 & 3.6 & 0.0 & -5.4 & $3.7 \times 10^{1}$ & $1.3 \times 10^{3}$ & $104446.07-595816.8$ & $8 \quad 12.6$ & 1.5 & -14.9 \\
\hline 694 & 1.5 & 1.5 & 3.6 & 0.2 & -5.5 & $4.0 \times 10^{1}$ & $1.3 \times 10^{5}$ & $104450.81-594442.9$ & 958.2 & 1.6 & -14.2 \\
\hline 768 & 2.6 & 1.4 & 3.7 & 0.1 & -5.9 & $1.1 \times 10^{2}$ & $4.6 \times 10^{2}$ & $104505.75-594405.0$ & $0 \quad 16.4$ & 1.3 & -14.7 \\
\hline 795 & 1.3 & 0.9 & 3.7 & 0.2 & -6.2 & $4.3 \times 10^{0}$ & $2.4 \times 10^{0}$ & $104511.09-594533.5$ & 566.4 & 1.4 & -14.1 \\
\hline 890 & 0.7 & 0.9 & 3.6 & 0.2 & -5.8 & $5.9 \times 10^{0}$ & $1.4 \times 10^{3}$ & $104529.17-601357.0$ & $0 \quad 20.7$ & 1.4 & -14.5 \\
\hline 928 & 1.0 & 0.8 & 3.6 & 0.1 & -6.0 & $9.7 \times 10^{0}$ & $3.0 \times 10^{2}$ & $104536.94-595611.3$ & 39.5 & 2.0 & -14.6 \\
\hline 987 & 0.6 & 0.7 & 3.5 & 0.2 & -5.3 & $3.3 \times 10^{1}$ & $9.1 \times 10^{2}$ & $104546.73-600343.3$ & 314.7 & 0.9 & -15.1 \\
\hline $1025^{\mathrm{c}}$ & 4.2 & 0.5 & 3.7 & 0.0 & -5.3 & $5.1 \times 10^{1}$ & $6.2 \times 10^{1}$ & $104553.71-595703.9$ & 978.1 & 1.5 & -13.8 \\
\hline 1055 & 3.6 & 2.8 & 4.0 & 0.6 & -5.2 & $7.7 \times 10^{0}$ & $5.1 \times 10^{0}$ & 104559.44-600517.9 & 9352.0 & 1.6 & -13.2 \\
\hline 1141 & 2.5 & 0.8 & 3.7 & 0.1 & -5.8 & $1.2 \times 10^{1}$ & $1.8 \times 10^{1}$ & $104622.54-594523.9$ & $9 \quad 14.2$ & 1.7 & -14.3 \\
\hline 1193 & 0.5 & 0.7 & 3.5 & 0.2 & -5.8 & $4.0 \times 10^{0}$ & $1.0 \times 10^{3}$ & $104642.52-600826.7$ & $7 \quad 7.9$ & 1.5 & -14.9 \\
\hline 1204 & 1.3 & 0.7 & 3.6 & 0.1 & -5.5 & $4.4 \times 10^{1}$ & $1.8 \times 10^{3}$ & $104646.68-600932.3$ & 34.0 & 1.4 & -14.9 \\
\hline 1276 & 0.8 & 0.8 & 3.6 & 0.1 & -8.1 & $7.7 \times 10^{0}$ & $2.8 \times 10^{1}$ & $104717.42-600906.1$ & 161.0 & 1.6 & -13.9 \\
\hline 1390 & 0.4 & 0.6 & 3.5 & 0.1 & -5.9 & $4.0 \times 10^{0}$ & $2.6 \times 10^{2}$ & $104836.21-601408.7$ & $\begin{array}{ll}7 & 7.7\end{array}$ & 1.3 & -14.9 \\
\hline
\end{tabular}

a The probability distributions of $A_{V, t}$ can be strongly skewed, with long tails to high values. This often yields very large values for $\sigma\left(A_{V, t}\right)$, hence in cases where $\sigma\left(A_{V, t}\right) \gg\left\langle A_{V, t}\right\rangle$ we define $\operatorname{Max}\left(A_{V, t}\right) \equiv \sigma\left(A_{V, t}\right)$.

$\mathrm{b}$ Columns (10)-(12) list the quantities NetCounts , EnergyFlux $_{\mathrm{t}}$, and MedianEnergy from Table 1 of Broos et al. (2011a).

c PCYC 1025 is located in the the very crowded center of the Treasure Chest, hence it is very likely a combination of multiple confused sources in the IRAC beam, and the physical association of the CCCP source with the IRAC source is highly questionable. 